\title{
Hippocampal Mossy Fibers Synapses in CA3 Pyramidal Cells Are Altered at an Early Stage in a Mouse Model of Alzheimer's Disease
}

\author{
(1)Silvia Viana da Silva, ${ }^{1,2 \star}$ Pei Zhang, ${ }^{1 *}$ @Matthias Georg Haberl, ${ }^{3}$ Virginie Labrousse, $\dagger^{1}$ Noëlle Grosjean, ${ }^{1}$ \\ Christophe Blanchet, ${ }^{1}$ Andreas Frick, ${ }^{3}$ and $\mathbb{C C h}^{2}$ ristophe Mulle ${ }^{1}$ \\ ${ }^{1}$ University of Bordeaux, Interdisciplinary Institute for Neuroscience, CNRS UMR 5297, F-33000 Bordeaux, France, ${ }^{2}$ BEB PhD Program CNC Coimbra, \\ 3030-789 Coimbra, Portugal, and ${ }^{3}$ University of Bordeaux, INSERM U1215, Neurocentre Magendie, 33077 Bordeaux, Cedex, France
}

Early Alzheimer's disease (AD) affects the brain non-uniformly, causing hippocampal memory deficits long before wide-spread brain degeneration becomes evident. Here we addressed whether mossy fiber inputs from the dentate gyrus onto CA3 principal cells are affected in an $\mathrm{AD}$ mouse model before amyloid $\beta$ plaque deposition. We recorded from CA3 pyramidal cells in a slice preparation from 6-month-old male APP/PS1 mice, and studied synaptic properties and intrinsic excitability. In parallel we performed a morphometric analysis of mossy fiber synapses following viral based labeling and 3D-reconstruction. We found that the basal structural and functional properties as well as presynaptic short-term plasticity at mossy fiber synapses are unaltered at 6 months in APP/PS1 mice. However, transient potentiation of synaptic transmission mediated by activity-dependent release of lipids was abolished. Whereas the presynaptic form of mossy fiber long-term potentiation (LTP) was not affected, the postsynaptic LTP of NMDAR-EPSCs was reduced. In addition, we also report an impairment in feedforward inhibition in CA3 pyramidal cells. This study, together with our previous work describing deficits at CA3-CA3 synapses, provides evidence that early AD affects synapses in a projection-dependent manner at the level of a single neuronal population.

Key words: Alzheimer; CA3; hippocampus; mossy fiber; mouse; synapse

Significance Statement

Because loss of episodic memory is considered the cognitive hallmark of Alzheimer's disease (AD), it is important to study whether synaptic circuits involved in the encoding of episodic memory are compromised in AD mouse models. Here we probe alterations in the synaptic connections between the dentate gyrus and CA3, which are thought to be critical for enabling episodic memories to be formed and stored in CA3. We found that forms of synaptic plasticity specific to these synaptic connections are markedly impaired at an early stage in a mouse model of $\mathrm{AD}$, before deposition of $\beta$ amyloid plaques. Together with previous work describing deficits at CA3-CA3 synapses, we provide evidence that early AD affects synapses in an input-dependent manner within a single neuronal population.

\section{Introduction}

At present no treatment is available to cure or stop the progression of Alzheimer's disease (AD). There is an urgent need for a

Received Nov. 8, 2018; revised Feb. 20, 2019; accepted March 8, 2019.

Author contributions: S.V.d.S., A.F., and C.M. designed research; S.V.d.S., P.Z., M.G.H., N.G., and V.L. performed research; S.V.d.S., P.Z., M.G.H., N.G., C.B., and C.M. analyzed data; S.V.d.S., P.Z., and C.M. wrote the first draft of the paper; S.V.d.S., P.Z., and C.M. wrote the paper; M.G.H. and C.B. contributed unpublished reagents/analytic tools; M.G.H., C.B., A.F., and C.M. edited the paper.

This work was supported by the Centre National de la Recherche Scientifique, by the ANR Grant SynFIAD, by a Grant from the Fondation France Alzheimer, by the Fundação para a Ciência e a Tecnologia, and by the Erasmus Mundus program "European Neuroscience Campus" from the 7th framework program. Cellular imaging was performed at the Bordeaux Imaging Center, a service unit of the CNRS-INSERM and Bordeaux University, member of the national infrastructure France Biolmaging. We thank Nelson Rebola and Mario Carta for their help with electrophys- better mechanistic understanding of the disease to develop improved therapeutic strategies, ideally at early disease stages. At the molecular level $\beta$-amyloid peptide $(\mathrm{A} \beta)$ deposition and tau ag-

iology and their constant scientific support, Klaus Conzelmann for providing rabies virus starter stocks, and Melanie Ginger and Katy Le Corf for their help with the virus production.

tWe dedicate this paper to the memory of our beloved friend and colleague Virginie Labrousse, date of death November 7, 2017.

The authors declare no competing financial interests.

*S.V.d.S. and P.Z. contributed equally to this work.

Correspondence should be addressed to Christophe Mulle at christophe.mulle@u-bordeaux.fr.

S. Viana da Silva's present address: Neurobiology Section and Center for Neuronal Circuits and Behavior, University of California, San Diego, La Jolla, CA 92093.

https://doi.org/10.1523/JNEUROSCI.2868-18.2019

Copyright $\odot 2019$ the authors 
gregation are known to cause senile plaques and intracellular neurofibrillary tangles. However, cognitive impairment in early AD strongly correlates with synaptic loss and dysfunction, rather than the aforementioned features or neuronal death (Selkoe, 2002). Loss of episodic memory is considered the cognitive hallmark of $\mathrm{AD}$ and deficits in episodic memory are already present in patients with mild cognitive impairment (MCI). The hippocampal region is critical for the acquisition of episodic memory and several studies show reduced activity in this region during memory tasks recorded in AD patients (Sperling, 2007). The CA3 circuits of the hippocampus are necessary for the early stages of memory acquisition, presumably by encoding instant representations of a context (Kesner and Rolls, 2015). Dentate gyrus (DG) and the CA3 region are essential for pattern separation and completion that are impaired in patients with MCI (Yassa et al., 2010). Notably, in contrast to patients with advanced $A D$, patients affected by MCI display hyperactivity in the DG and CA3 regions of the hippocampus during memory encoding, indicating a dysfunctional encoding mechanism (Yassa et al., 2010). It is thus important to study the physiopathological status of DG-CA3 synaptic circuits in models of $\mathrm{AD}$ to enhance our understanding of the cellular underpinnings of memory dysfunction.

CA3 principal cells (PCs) receive three main types of glutamatergic inputs: recurrent CA3 collaterals [associational/commissural (A/C) fibers], entorhinal cortex projections [perforant path (PP)] and mossy fiber (Mf) inputs from DG granule cells. These inputs are precisely positioned along the apical and basal dendrites, and greatly vary in their structural and functional properties (Rebola et al., 2017). Behaviorally, A/C synapses are thought to be essential for short-term memory, whereas Mf synapses and PP synapses would be required for the acquisition and retrieval of contextual memories, respectively (Rolls, 2013). In a contextual fear conditioning paradigm, the DG plays a role in both fear acquisition and extinction (Bernier et al., 2017), suggesting that the function and plasticity of the projection from DG granule cells to CA3 PCs via Mf synapses is required for both these processes. Despite the known role of DG in pattern separation and in memory formation (Leutgeb et al., 2007), deficits in Mf-CA3 PCs in models of $\mathrm{AD}$ are poorly understood (Witton et al., 2010; Marchetti and Marie, 2011).

We recently demonstrated that long-term synaptic potentiation (LTP) within the associative network is abolished in CA3 PCs at an early stage in the APP/PS1 mouse model of AD (Viana da Silva et al., 2016). Importantly, these mice were shown to be impaired in contextual fear conditioning (Kilgore et al., 2010). In contrast to A/C synapses, Mf-CA3 synapses display a wide dynamic range of short-term plasticity, and express presynaptic forms of LTD and LTP that are independent of NMDA receptor (NMDAR) activation (Nicoll and Schmitz, 2005; Rebola et al., 2017). In addition, at Mf-CA3 synapses, NMDARs are themselves subject to LTP (Kwon and Castillo, 2008a; Rebola et al., 2008). Finally, Mfs make synaptic contacts with GABAergic interneurons within CA3, thereby relaying robust feedforward inhibition of CA3 PCs (Torborg et al., 2010). This connection is amenable to structural plasticity upon memory encoding (Ruediger et al., 2011).

In this study, we investigated whether the structural and physiological properties of Mf-CA3 synapses were impaired in a mouse model of early AD. In addition, we tested whether feedforward inhibition via this pathway is altered in these mice, because it tightly controls the flow of information from DG to CA3 (Torborg et al., 2010; Zucca et al., 2017).

\section{Materials and Methods}

Mice. The APP/PS1 mice used express the amyloid precursor protein $(A P P)$ gene with the Swedish mutations (KM670/671NL) and the presenilin 1 (PSEN1) gene with a deletion of exon 9 (Jankowsky et al., 2004; Garcia-Alloza et al., 2006). Mice were obtained from The Jackson Laboratory and used according to regulations of the University of Bordeaux/ CNRS Animal Care and Use Committee. Throughout their life, all mice were group-housed, ranging from 4 to 10 animals per cage. Food and water were provided ad libitum. The transparent Plexiglas cages $(38.1 \times$ $19.1 \times 12.7 \mathrm{~cm}$ ) were maintained on a $12 \mathrm{~h}$ dark/light cycle, kept in a temperature-regulated room, and protected from exterior pathogens by a filter. All experiments were performed in the light phase of the circadian cycle in 6 months (26-32 weeks) male APP/PS1 and age-matched male wild-type (WT) littermates.

Stereotaxic injections and confocal microscopy. Viral stocks were stored at $-80^{\circ} \mathrm{C}$ until use and were thawed on ice before their in vivo administration. Before surgery the mice were anesthetized by inhalation of isoflurane in an induction chamber connected to tubing that delivered a mix of air and $4 \%$ isoflurane. Once the animals were immobile they were placed on a heating $\mathrm{pad}\left(36^{\circ} \mathrm{C}\right)$ while they continued to receive anesthesia via a mask (1.5-2\% isoflurane) during the surgery. For labeling CA3 pyramidal neurons we injected the glycoprotein deleted rabies virus variant coated with the native glycoprotein (RVDG-eGFP RG, $300 \mathrm{nl}$, velocity $40 \mathrm{nl} / \mathrm{min}$ ) into the CA1 stratum radiatum (coordinates: anteroposterior: $-1.92 \mathrm{~mm}$; mediolateral: $\pm 1.50 \mathrm{~mm}$; and ventral: $-1.35 \mathrm{~mm}$ ) to achieve a retrograde infection. For labeling mossy fiber terminals we injected an anterograde variant of the glycoprotein deleted rabies virus (RVAG-tdTom VSVG, $500 \mathrm{nl}$, velocity $50 \mathrm{nl} / \mathrm{min}$; Haberl et al., 2015) into the hilus of DG (coordinates: anteroposterior: $-1.92 \mathrm{~mm}$; mediolateral: $\pm 1.15 \mathrm{~mm}$; and ventral: $-2.00 \mathrm{~mm}$ ). The mice were killed $6 \mathrm{~d}$ after the injection. Injected mice were anesthetized with intraperitoneal injection of pentobarbital $(30 \mathrm{mg} / \mathrm{kg})$, then transcardially perfused with Ringer's solution [135 mM NaCl, $5.4 \mathrm{~mm} \mathrm{KCl}, 1.8 \mathrm{~mm} \mathrm{CaCl}_{2}, 1 \mathrm{~mm}$ $\mathrm{MgCl}_{2}, 5 \mathrm{~mm}$ HEPES, pH 7.4 with heparin (1:1000), followed by $4 \%$ paraformaldehyde (PFA)]. Brains were removed and postfixed overnight in $4 \%$ PFA. After fixation, coronal sections $(70 \mu \mathrm{m})$ were cut on a vibratome (Leica), and then mounted on a glass slide and covered with a thin glass coverslip. To analyze the morphology of Mf-CA3 synapses we used a confocal microscope (SP5, Leica Microsystems) equipped with an Argon 488 Laser, to acquire $Z$-stacks at randomly chosen positions in the stratum lucidum of CA3b. Images were obtained with a $63 \times$ objective (numerical aperture, NA 1.4) and regular photomultipliers.

Morphology analysis. The $Z$-stacks were analyzed with IMARIS software (Bitplane) performing 3D volume reconstructions of the $\mathrm{Mf}$ boutons (MfBs) and thorny excrescences (ThEs) to obtain, in a semiautomatic way, the values of volume and surface area for each structure, as well as the number of filopodia. Calculation of the complexity index was done as previously described (Bednarek and Caroni, 2011). Briefly, we calculated the maximum volume $\left(V_{\mathrm{Max}}\right)$ the bouton could span given the measured surface (a sphere) and divide it by the real measured bouton volume $\left(V_{\text {Bouton }}\right)$. Only cells with a complete $\mathrm{ThE}$ within the $70 \mu \mathrm{m}$ slices were reconstructed and analyzed.

Slice preparation. Anesthesia drug (ketamine $75 \mathrm{mg} / \mathrm{kg}$, xylazine 10 $\mathrm{mg} / \mathrm{kg}$ ) was diluted in saline and injected intraperitoneally to the mouse 5 min before decapitation or transcardial perfusion followed by decapitation. The head was immediately placed into a Petri dish filled with ice-cold cutting solution (in mM: 200 sucrose, 20 glucose, $0.4 \mathrm{CaCl}_{2}, 8$ $\mathrm{MgCl}_{2}, 2 \mathrm{KCl}, 1.3 \mathrm{NaH}_{2} \mathrm{PO}_{4}, 26 \mathrm{NaHCO}_{3}, 1.3$ ascorbate, 0.4 pyruvate, and 3 kynurenic acid, $\mathrm{pH} 7.3)$ oxygenated with carbogen $\left(95 \% \mathrm{O}_{2}, 5 \%\right.$ $\mathrm{CO}_{2}$ ). The brain was rapidly removed from the scull and parasagittal slices $(350 \mathrm{~mm})$ were cut with a Leica vibratome (VT 1200S) in the cutting solution. The slices were then kept at $33^{\circ} \mathrm{C}$ in oxygenated resting solution (in mm: $110 \mathrm{NaCl}, 2.5 \mathrm{KCl}, 0.8 \mathrm{CaCl}_{2}, 8 \mathrm{MgCl}_{2}, 1.25 \mathrm{NaH}_{2} \mathrm{PO}_{4}$, $26 \mathrm{NaHCO}_{3}, 0.4$ ascorbate, 3 pyruvate, and 14 glucose, $\mathrm{pH} 7.3$ ) for 20 $\mathrm{min}$, before being transferred to aCSF. The slices were then left at room temperature for a maximum of $6 \mathrm{~h}$ after cutting.

Electrophysiological recordings. The recording chamber of the electrophysiology setup was perfused with oxygenated aCSF (in mM: $125 \mathrm{NaCl}$, 
$2.5 \mathrm{KCl}, 2.3 \mathrm{CaCl}_{2}, 1.3 \mathrm{MgCl}_{2}, 1.25 \mathrm{NaH}_{2} \mathrm{PO}_{4}, 26 \mathrm{NaHCO}_{3}$, and 14 glucose, $\mathrm{pH}$ 7.4). $\mathrm{CA} 3$ pyramidal neurons in the $\mathrm{CA} 3 \mathrm{~b}$ subregion were identified by differential interference contrast microscopy using an Olympus fixed stage upright microscope (BX51WI) equipped with a $60 \times$ magnification immersion objective at room temperature, and whole-cell patch-clamp configuration was achieved with borosilicate glass capillaries with resistance value ranging from 3 to $5 \mathrm{M} \Omega$. The pipettes were filled with variant intracellular solutions depending on experiments: $\mathrm{KMSO}_{3}$-based solution (in mM: $120 \mathrm{KMSO}_{3}, 2 \mathrm{MgCl}_{2}, 1$ $\mathrm{CaCl}_{2}, 20 \mathrm{KCl}, 10 \mathrm{EGTA}, 2 \mathrm{ATPNa}$, and 10 HEPES, pH 7.2) was used for current-clamp recordings, CsCl-based solution (in mM: $120 \mathrm{CsCl}, 2$ $\mathrm{MgCl}_{2}, 2 \mathrm{CaCl}_{2}, 5 \mathrm{EGTA}, 5$ phosphocreatine, $2 \mathrm{ATPNa}_{2}, 0.33 \mathrm{GTP}, 10$ HEPES, and 10 QX314, pH 7.2) was used for IPSC recordings, and $\mathrm{CsMSO}_{3}$-based solution (in mm: $100 \mathrm{CsMSO}_{3}, 3 \mathrm{MgSO}_{4}, 3.5 \mathrm{CaCl}_{2}, 20$ EGTA, 5 phosphocreatine, $3 \mathrm{ATPNa}_{2}, 0.33$ GTP, and 10 HEPES, pH 7.2) was used for all other experiments. Cells were allowed to stabilize for $8-10$ min after whole-cell configuration was established. To monitor the access resistance during the whole recording time, a hyperpolarizing voltage step $(-5 \mathrm{mV}, 10 \mathrm{~ms})$ was applied at the beginning of each trace. Series access resistance was $<20 \mathrm{M} \Omega$, and when it changed by $>20 \%$, the recording was discarded. When held at $-70 \mathrm{mV}$, neurons with a holding current $>250 \mathrm{pA}$ were also rejected. Mf synaptic currents were identified according to the following criteria: (1) obvious paired-pulse facilitation (PPF), (2) EPSCs had a steep rising phase ( $\sim 1 \mathrm{~ms})$, and (3) EPSCs decays free of secondary peaks that might indicate the presence of polysynaptic contamination. Liquid junction potential correction was not used for measurements of membrane potentials. Unless stated differently all baselines were established with a stimulation at $0.1 \mathrm{~Hz}$ and compared with the same stimulation frequency after a drug application or stimulation protocol.

Recordings were made using an EPC10.0 amplifier (HEKA Elektronik), filtered at $0.5-1 \mathrm{kHz}$ and analyzed using IGOR Pro and Neuromatic v2.6 software. All drugs for electrophysiological experiments were obtained from Tocris Biosciences or Sigma-Aldrich, unless otherwise stated.

Experimental design and statistical analysis. Statistical analyses were performed with Prism6 (GraphPad Software). First the normality of dataset was tested using the D'Agostino-Pearson omnibus normality test. If data were normally distributed, a Student's $t$ test, one-way ANOVA or two-way ANOVA was performed; otherwise, nonparametric tests such as Mann-Whitney test (for unpaired data) were used. Data distributions were analyzed using the Kolmogorov-Smirnov (KS) test; for miniature events the distribution curve was calculated for each cell and all cells averaged per condition to create a single curve. For electrophysiological data, the $n$ values can be found in the figure legends and correspond to the number of cells analyzed (the number of mice used is also reported). Only one recording per slice was performed. Results were presented as mean \pm SEM unless stated otherwise. Statistical differences were considered significant at $p<0.05$.

\section{Results}

Morphometric analysis of Mf-CA3 synapses in APP/PS1 mice reveals subtle alterations in pre and postsynaptic compartments

Mf synapses are subject to robust structural rearrangement following learning and experience-induced synaptic plasticity (Galimberti et al., 2006; Caroni et al., 2012). This prompted us to assess possible morphological changes in Mf synapses in our model of AD. To evaluate structural changes at Mf-CA3 synapses at an early phase of AD, we used APP/PS1 mice at 6 months of age, before observable plaque deposits in the hippocampus (Viana da Silva et al., 2016). In contrast to A/C and PP synapses which are of the single site/single spine type, Mf inputs make synaptic contacts on proximal dendrites of CA3 PCs in the stratum lucidum via "giant" MfBs with multiple glutamate release sites facing large postsynaptic structures called ThEs (Amaral and Dent, 1981). We probed changes in morphological properties of
Mf-CA3 synapses both at the presynaptic and postsynaptic site. To label both MfBs and ThEs, an anterograde and a retrograde version of recombinant rabies viruses (RV; Haberl et al., 2017) were stereotaxically injected into the DG and CA1 region, respectively. MfBs (expressing td-Tomato) were reconstructed from confocal $Z$-stacks obtained from the CA3b subregion (Fig. 1A). We focused on the hippocampal CA3b subregion which may play an important role in associative memory recall and pattern completion (Kesner, 2013; Sun et al., 2017). The mean surface area of MfBs did not differ between WT $\left(67.1 \pm 1.8 \mu \mathrm{m}^{2}\right)$ and APP/PS1 mice $\left(67.9 \pm 1.6 \mu \mathrm{m}^{2}\right.$; Fig. $\left.1 B\right)$. Similarly, the distribution and mean volume values of MfBs did not show any statistical difference $\left(15.2 \pm 0.4 \mu \mathrm{m}^{3}\right.$ in WT mice and $14.6 \pm 0.4 \mu \mathrm{m}^{3}$ in APP/ PS1 mice; Fig. $1 C)$.

MfBs contact not only CA3-PCs, but also inhibitory interneurons in the hilus and stratum lucidum within CA3, via filopodial protrusions emerging from the MfBs (Acsády et al., 1998). To estimate the number of filopodia as a readout for the innervation density of interneuron activation, we compared the complexity index of the reconstructed MfBs (see Materials and Methods). This index takes into account the fact that these structures encompass a tiny volume but a considerable membrane area. Although the average values of the complexity index did not differ between the genotypes, a significant difference was observed when analyzing the cumulative distribution of MfB complexity indexes (KS test, $p<0.0001$ ). To further analyze the difference in complexity we divided MfBs into three categories based on their volume: small $\left(<10 \mu \mathrm{m}^{3}\right)$, medium $\left(10-20 \mu \mathrm{m}^{3}\right)$, and large $\left(>20 \mu \mathrm{m}^{3}\right)$. We observed a significant increase in complexity index for the large MfBs in APP/PS1mice (4.8 \pm 0.1 ) compared with WT littermates $(4.4 \pm 0.1$, one-way ANOVA $p<0.0001$; Fig. 1D). An increase in the complexity index may indicate an increase in the number and/or length of MfB filopodia. A direct count of filopodia revealed that APP/PS1 mice display an increase in their number per MfB compared with WT mice (APP/PS1 $1.0 \pm 0.03$, WT $0.5 \pm 0.04$, Mann-Whitney test, $p<0.0001$; Fig. 1E).

To study the postsynaptic ThEs, a retrograde RV-expressing GFP was used to infect CA3 PCs from their axon terminals in CA1 (Fig. $1 F$ ). Reconstructions were performed only from ThEs located in the stratum lucidum; ThEs located on basal dendrites of CA3 PCs were not included in the study. The number of ThEs per cell was not different between the two genotypes (WT $58.2 \pm 4.0$ and APP/PS1 $51.6 \pm 5.8$; Fig. $1 G$ ). We found a significant difference in the distribution of ThE surface values toward smaller surface areas in the APP/PS1 mice (KS test, $p<0.0001$ ), although there was no significant difference in the mean surface values (WT $16.6 \pm 0.6 \mu \mathrm{m}^{2}$, APP/PS1 $14.2 \mu \mathrm{m}^{2} \pm 0.5 \mu \mathrm{m}^{2}$; Fig. $1 H$ ) Similarly, we found no alteration of the mean volume of ThEs in APP/PS1 mice (WT $2.4 \pm 0.1 \mu \mathrm{m}^{3}$, APP/PS1 $2.0 \pm 0.1$ $\mu \mathrm{m}^{3}$ ) but a significant shift toward smaller ThEs volumes in the distribution analysis (KS test, $p<0.0001$; Fig. 1I). The summary plot of the surface and volume of each reconstructed ThE (Fig. 1J) shows that the relationship between these two parameters is not altered in 6-month-old APP/PS1 mice (WT linear regression slope $5.72 \pm 0.04$; APP/PS1 linear regression slope $5.60 \pm 0.06)$. Overall, careful morphometric examination of several parameters of Mf synapses showed that in addition to subtle alterations of the presynaptic and postsynaptic compartments, the number of filopodia per MfB was doubled in APP/PS1 mice. 
A
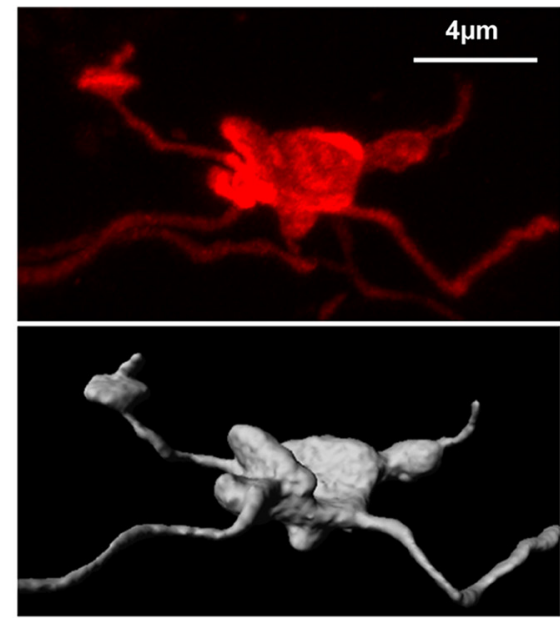

F

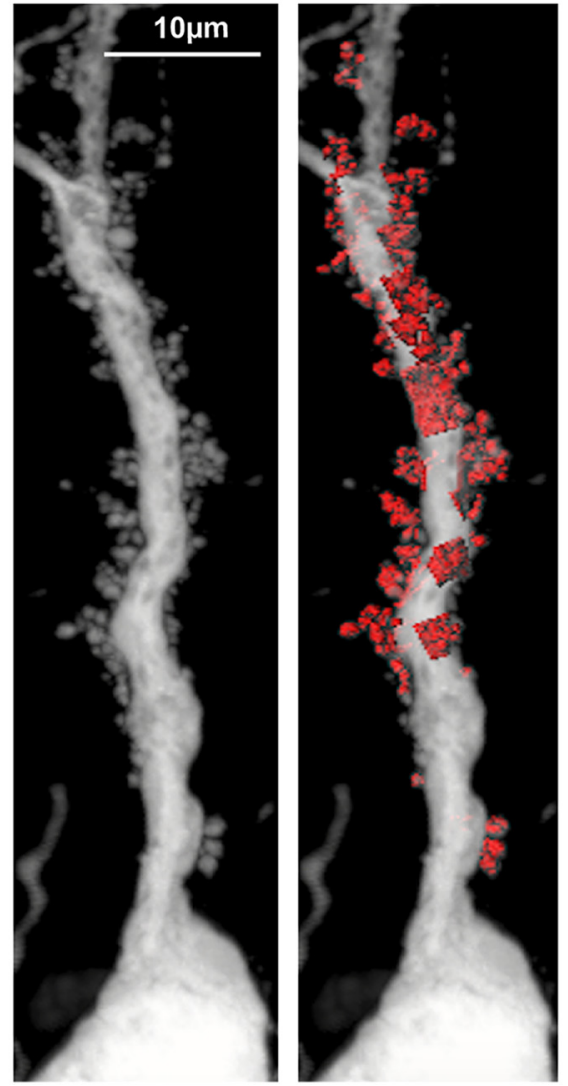

B

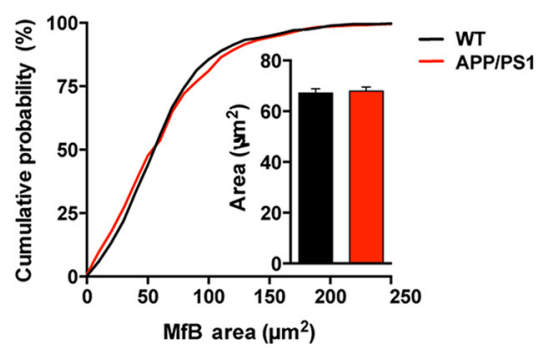

D

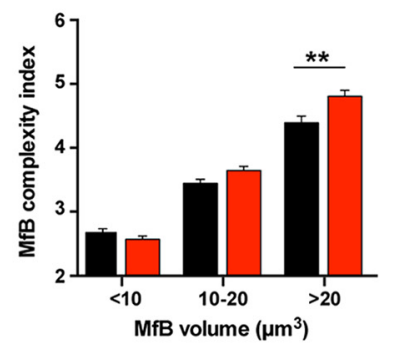

G

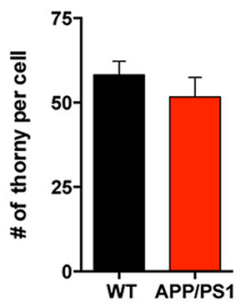

I

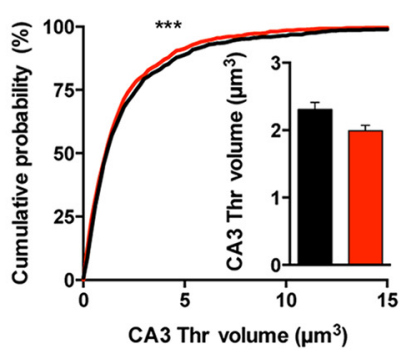

C

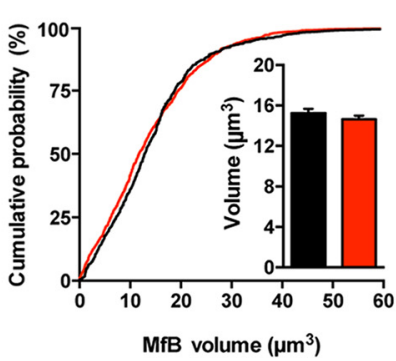

E

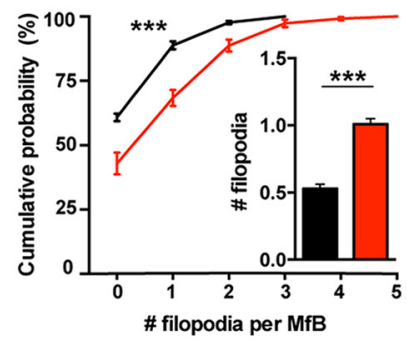

H

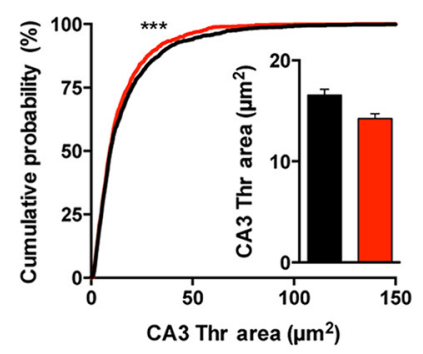

J

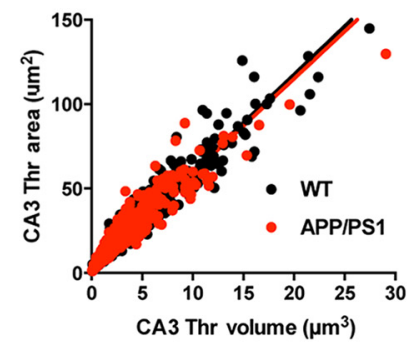

Figure 1. Morphology of the MfBs and postsynaptic thorny excrescences of CA3 PCs in APP/PS1 mice. A, Example image showing a maximum intensity projection of an MfB acquired with confocal microscopy with the representative 3D volumetric reconstruction. $B$, Cumulative distribution of the area of MfB reconstructed from WT ( $n=609,5$ mice) and APP/PS1 mice ( $n=868,6$ mice) are not statistically different (KS test, $p=0.1440$ ). Mean values of area are also not significantly different (Mann-Whitney test, $p=0.6624)$. C, Cumulative distribution of the volume from reconstructed MfBs from WT ( $n=609,5$ mice) and APP/PS1 mice $(n=868,5$ mice; KS test, $p>0.999)$. Mean values of MfB volume are not significantly different (Mann-Whitney test, $p=0.3116)$. $D$, Distribution of the complexity index calculated from MfB from WT ( $n=609$ boutons, 5 mice) and APP/PS1 mice $(n=868$ boutons, 5 mice). The distribution of values is significantly different (KS test, $p<0.0001$ ). We analyzed separately the complexity index for three classes of MfBs sizes found in the (A3b region. Smaller MfB have a volume $<10 \mu \mathrm{m}^{3}$, medium MfBs have a volume ranging from 10 to $20 \mu \mathrm{m}^{3}$, and large MfBs have volume values $>20 \mu \mathrm{m}^{3}$. We found a group difference in the complexity index of APP/PS1 MfB (one-way ANOVA, $p<0.0001$ ). For small MfBs the complexity index is $2.67 \pm 0.06$ for WT mice $(n=202,33.2 \%)$ and $2.57 \pm 0.05$ for APP/PS1 mice $(n=337,37.6 \%)$, displaying no significant difference between genotypes (Sidak's multiple-comparisons test, $p=0.6388)$. Similarly, medium size MfBs are not statistically different between WT ( $3.44 \pm 0.06, n=266,43.8 \%)$ and APP/PS1 mice (3.65 $\pm 0.06, n=331,36.9 \%$; Sidak's multiple-comparisons test, $p=0.0713)$. Whereas the mean complexity index of the large MfBs was increased in APP/PS1 mice $(4.81 \pm 0.09, n=228,25 \%)$ compared with WT mice $(4.39 \pm$ $0.06, n=140,25.5 \%$; Sidak's multiple-comparisons test, $p=0.0014$ ). $E$, Cumulative distribution of the number of filopodia per MfB (APP/PS1 $n=868,6$ mice; WT $n=609,5$ mice; $K S$ test, $p<$ 0.0001 ) shows an increase for APP/PS1 mice (Mann-Whitney test, $p<0.0001$ ). $F$, Example image showing a maximum intensity projection of the proximal dendrite of a CA3b pyramidal cell infected by RV $\Delta$ G-eGFP with the native envelope acquired with confocal microscopy and subsequent reconstruction of ThE in stratum lucidum. $G$, Average total number of ThE per pyramidal cell; just ThE on apical dendrites were analyzed and only cells with complete apical dendritic arborization were used. The number of ThE is not statistically different between APP/PS1 $(n=8$ complete cells, 4 mice) and WT mice (WT $n=9$ complete cells, 4 mice; unpaired $t$ test, $p=0.3539$ ). $\boldsymbol{H}$, Cumulative distribution of the area from reconstructed ThE shows a difference between WT ( $n=1259$, 4 mice) and APP/PS1 mice ( $n=945,4$ mice; KS test, $p<0.0001)$. But there is no significant difference regarding the mean surface area of reconstructed ThE in the two genotypes (WT $n=1259,4$ mice; APP/PS1, $n=945,4$ mice; Mann-Whitney test, $p=0.1074$ ). I, Cumulative distribution of reconstructed ThE volume shows that there is a small difference in the distribution of ThE between WT ( $n=1259,4$ mice) and APP/PS1 mice $(n=945,4$ mice; KS test, $p<0.0001)$. Nevertheless, the mean volume values in the APP/PS1 mice is not statistically different from those in the WT littermates (Mann-Whitney test, $p=0.1221)$.J, Summary plot of the relation between surface area and volume of CA3 ThE. There is no difference in the ratio of surface area to volume between the WT $\left(r^{2}=0.937\right)$ and APP/PS1 mice $\left(r^{2}=\right.$ 0.910 ) because slopes values are very similar (WT slope is $5.72 \pm 0.04$ and APP/PS1 slope is $5.60 \pm 0.057$; linear regression, $p=0.0941$ ). All data represented as mean \pm SEM. ${ }^{* *} p<0.01,{ }^{* * *} p<0.001$. 
A

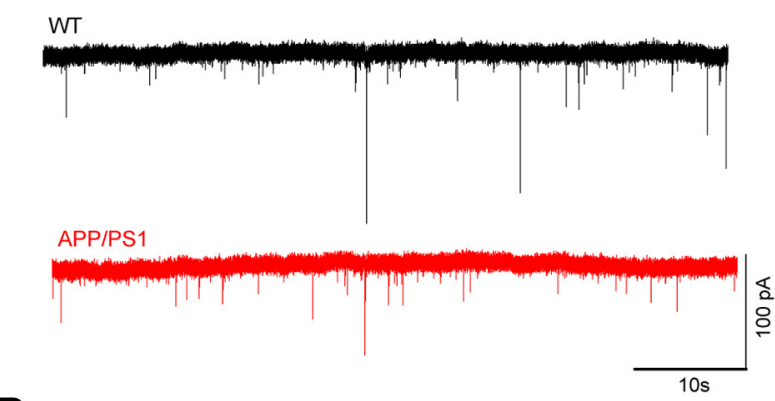

D
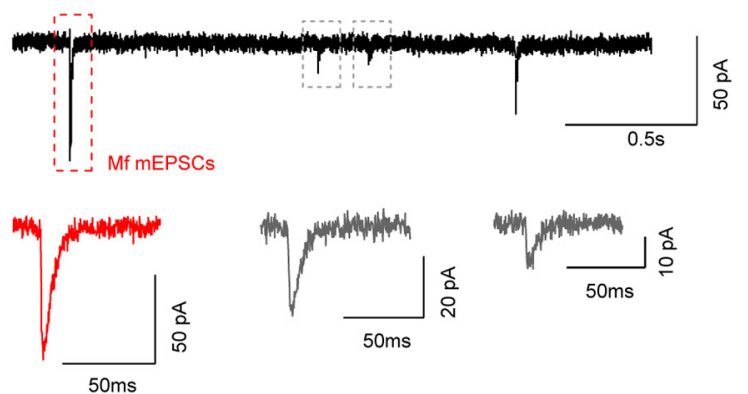

B

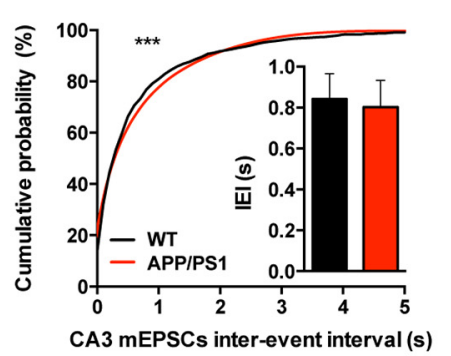

E

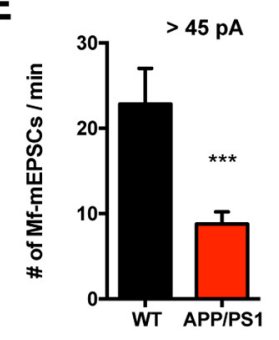

$F$
C
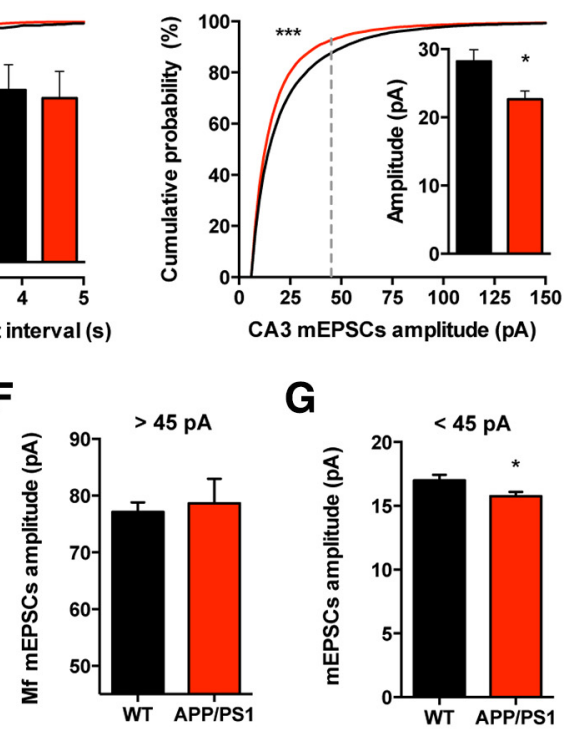

G

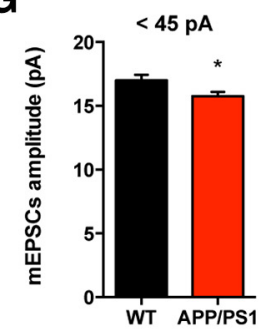

Figure 2. Miniature EPSCs arising from Mf synapses are not altered in APP/PS1 mice. $A$, Representative recordings of mEPSCs obtained from CA3 PCs from WT and APP/PS1 mice. CA3 PCs were held at $-70 \mathrm{mV}$ in the presence of bicuculline $(10 \mu \mathrm{m})$ and TTX (1 mM). All the events occurred during the 15 min recordings were analyzed and averaged per cell (WT $n=15$ cells, 8 mice; APP/PS1 $n=15$ cells, 9 mice). $\boldsymbol{B}$, Cumulative probability analysis of mEPSC IEls shows a significant difference between WT and APP/PS1 mice (KS test, $p<0.0001$ ) but the average frequency of mEPSC is not statistically different between WT and APP/PS1 mice (Mann-Whitney test, $p=0.8146$ ). C, Cumulative probability analysis of mEPSCs amplitudes showed a difference between WT and APP/PS1 distributions (KS test, $p<0.0001$ ); the average amplitude of mEPSCs is decreased in APP/PS1 mice (Mann-Whitney test, $p=0.035$ ). Gray dashed line represents the border between Mf-driven events $(\boldsymbol{D}-\boldsymbol{F})$. $\boldsymbol{D}$, Example of putative Mf-mEPSC (red framed event): all peaks with amplitudes $>45 \mathrm{pA}$ were considered as most likely Mf-driven. $\boldsymbol{E}$, Number of events with amplitudes $>45 \mathrm{pA}$ per minute. On average, APP/PS1 mice show a significant decrease in the number of mEPSCs events larger than $45 \mathrm{pA}$ (Mann-Whitney test, $p<0.0001$ ). $\boldsymbol{F}$, Average amplitude of mEPSCs larger than $45 \mathrm{pA}$ is not statistically different between genotypes; Mann-Whitney test, $p=0.3627$. G, Small mEPSCs events ( $<45 \mathrm{pA}$ ) show a subtle but significant decrease in average amplitude in APP/PS1 mice, unpaired $t$ test, $p=0.0322$. All data presented as mean \pm SEM. ${ }^{*} p<0.05,{ }^{* * *} p<0.001$.

\section{Basal synaptic properties and presynaptic plasticity are} unaltered at Mf-CA3 synapses in APP/PS1 mice

The density of dendritic spines is correlated with the number of glutamatergic synapses. To understand whether the number and properties of glutamatergic synapses are generally altered in preplaque APP/PS1 mice we recorded spontaneous miniature EPSCs (mEPSCs) from CA3b PCs.

Synaptic activity was recorded for $15 \mathrm{~min}$ in the presence of the $\mathrm{GABA}_{\mathrm{A}}$ receptor blocker, bicuculline $10 \mu \mathrm{M}$ ), to isolate glutamatergic inputs (Fig. $2 A$ ). The inter-event interval (IEI) distribution of the mEPSCs was statistically different between genotypes (KS test, $p<0.0001$ ), whereas the IEI mean value was not (APP/PS1 mice: $0.80 \pm 0.1 \mathrm{~s}$; WT: $0.84 \pm 0.12 \mathrm{~s}$; Fig. $2 B)$. The mean amplitude of all events that occurred during a 15 min recording period was significantly smaller in APP/PS1 mice (22.6 $1.2 \mathrm{pA})$ compared with WT $(28.2 \pm 1.7 \mathrm{pA}$, Mann-Whitney test, $p=0.035$ ), resulting in an altered distribution of mEPSC amplitudes between genotypes (KS test, $p<0.0001$; Fig. $2 C$ ). Previous studies have shown that elimination of DG cells by $\gamma$-irradiation results in the loss of mEPSCs with an amplitude $>45 \mathrm{pA}$ (Henze et al., 1997), suggesting that these events are most likely Mfdriven, whereas smaller events might represent any of the three main glutamatergic inputs into CA3 PCs (Henze et al., 1997). In our recordings mEPSCs larger than $45 \mathrm{pA}$ were much less frequent in the APP/PS1 CA3 PCs $(8.7 \pm 1.4 \%$ of total mEPSCs $)$ compared with WT $(22.8 \pm 4.2 \%$, Mann-Whitney test, $p<$ 0.0001 ; Fig. $2 D, E)$, suggesting a decrease in the number of Mfdriven events. We analyzed the amplitude of Mf-events (i.e., $>45$ $\mathrm{pA}$ ) and found no difference in the average amplitude between genotypes (WT $77.1 \pm 1.7 \mathrm{pA}$ and APP/PS1 $78.7 \pm 4.3$ pA; Fig.
$2 F)$. When analyzing the remaining events $(<45 \mathrm{pA})$ we observed a decrease in their amplitude in the APP/PS1 mice (15.8 \pm $0.3 \mathrm{pA})$ compared with the WT controls $(17.0 \pm 0.4 \mathrm{pA}$, MannWhitney test, $p=0.0322$; Fig. $2 G$ ). This is in line with our previous study showing that $\mathrm{A} / \mathrm{C}$ synapses are affected in these mice (Viana da Silva et al., 2016).

We then recorded evoked Mf-CA3 EPSCs using minimal stimulation conditions (Marchal and Mulle, 2004). We first observed that the failure rates recorded at a stimulation frequency of $0.1 \mathrm{~Hz}$ in APP/PS1 mice (12.6 $\pm 3.3 \%$, average of 40 sweeps per cell) were not significantly different from the values recorded in WT littermates (16.5 $\pm 3.1 \%$; Fig. $3 A, B)$. Furthermore, the absolute amplitudes recorded in WT and APP/PS1 CA3 PCs were similar (WT 76.2 \pm 7.8 pA and APP/PS1 $80.0 \pm 15.4$ pA; Fig. $3 C$ ). Low-frequency facilitation is a characteristic presynaptic feature of Mf-CA3 PC synapses (Rebola et al., 2017), which can be observed when switching stimulation frequency from 0.1 to $1 \mathrm{~Hz}$. The mean value of facilitation was comparable between genotypes as illustrated in the representative traces in Figure $3 D$ (WT $6.1 \pm 0.5$ and APP/PS1 $5.5 \pm 0.3 ; t$ test, $p=0.4298$, average amplitude of 60 sweeps at $1 \mathrm{~Hz}$ divided by average amplitude of 40 sweeps at $0.1 \mathrm{~Hz}$; Fig. $3 D, E)$. We also observed that in 6-month-old WT mice the PPF ratio, in response to two temporally close afferent stimulations ( $40 \mathrm{~ms}$; WT 6 months: $2.65 \pm 0.19$, average of 20 sweeps per cell separated by $30 \mathrm{~s}$; Fig. $3 F, G$ ) was smaller than PPF ratio values obtained from 3- to 4-week-old WT mice (Pinheiro et al., 2007; Lanore et al., 2012). No alteration of this presynaptic feature was observed in 6-month-old APP/PS1 mice (APP/ PS1: $2.84 \pm 0.36$; Fig. $3 F, G)$. Finally, we found no difference between the genotypes in the facilitation of Mf-EPSCs in response to 
A

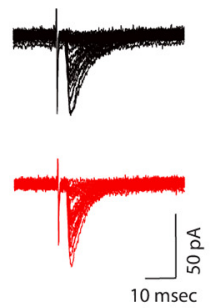

B

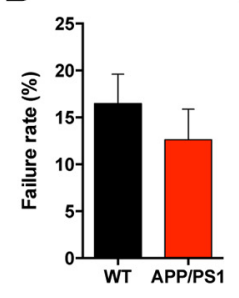

C

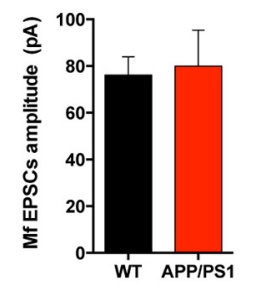

D

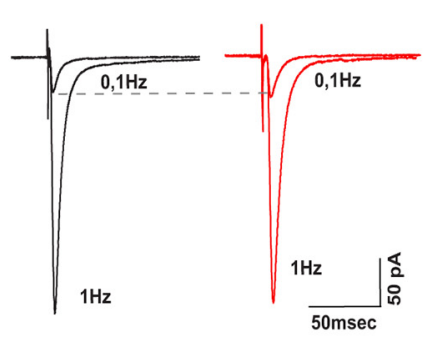

E

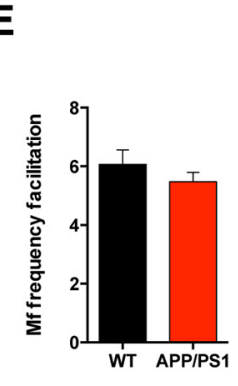

F

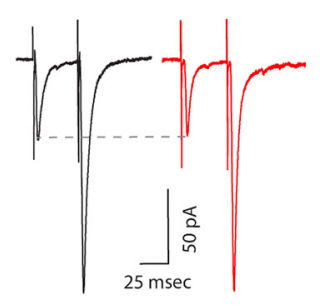

G

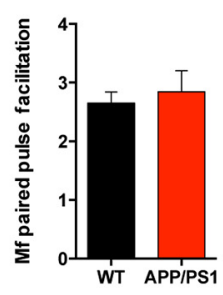

H
I
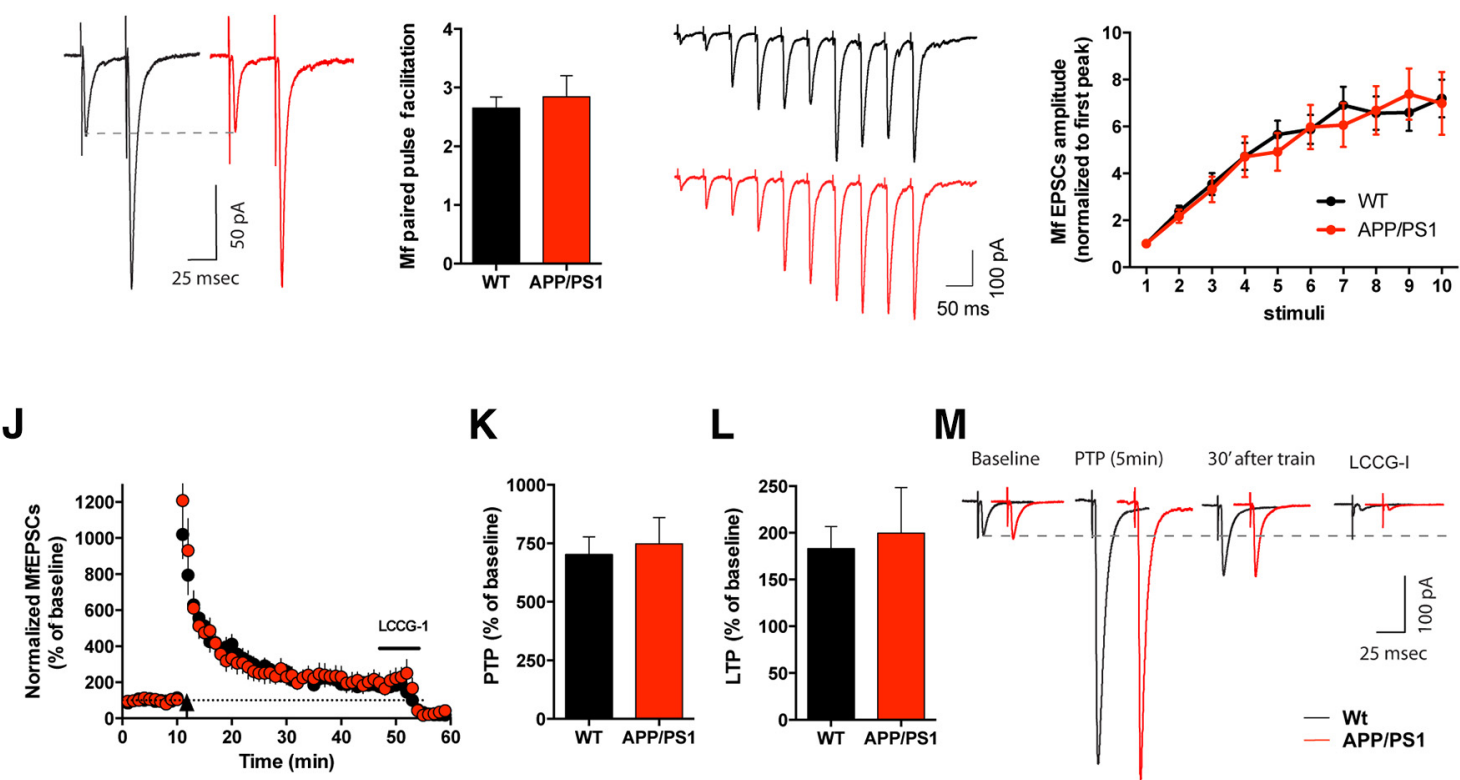

M

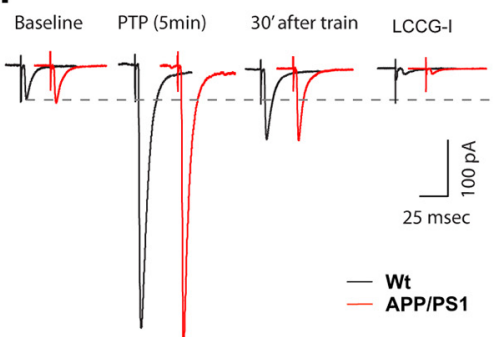

Figure 3. Presynaptic short-term plasticity and presynaptic LTP are normal at Mf-CA3 synapses of APP/PS1 mice. $A$, Representative recording from WT and APP/PS1 cell displaying the typical high variability of the Mf-EPSC amplitudes obtained at $0.1 \mathrm{~Hz}$, with several failures. $\boldsymbol{B}$, Failure rates show no significant difference between WT ( $n=15,10 \mathrm{mice}$ ) and APP/PS1 ( $n=19,13$ mice) cells, unpaired $t$ test, $p=0.4078$. Values are obtained for each genotype by calculating the percentage of failures at $0.1 \mathrm{~Hz}$ (events $<5 \mathrm{pA}$ were considered as noise). $C$, Average amplitude of Mf-EPSCS recorded at minimal stimulation conditions is not statistically different between WT ( $n=15,13$ mice) and APP/PS1 mice $(n=19,10$ mice; Mann-Whitney test, $p=0.678)$. D, Representative average traces of the amplitude increase of Mf-EPSCS with FF. EPSCs obtained at $0.1 \mathrm{~Hz}$ (30 sweeps) and $1 \mathrm{~Hz}$ (30 sweeps) for WT and APP/PS1 mice. E, Similar mean values of FF were observed in WT ( $n=18,15$ mice) and APP/PS1 ( $n=23,18$ mice; unpaired $t$ test, $p=0.0601)$. $F$, Average of 20 sweeps illustrating PPF at an interstimulus interval of $40 \mathrm{~ms}$ (inter-sweep interval $20 \mathrm{~s}$ ) for WT and APP/PS1 Mf synapses. G, Bar graph representing the ratio between the amplitude of the second over the first EPSCs obtained during paired pulse stimulating protocol. APP/PS1 PPF mean values ( $n=17,13$ mice) are not statistically different from WT ( $n=14,10$ mice; Mann-Whitney test, $p=0.7319)$. $\boldsymbol{H}$, Average of 5 traces illustrating 10 stimuli $20 \mathrm{~Hz}$ train at the interval of 2 min apart, for WT and APP/PS1 mice. I, Mf EPSC amplitude increases during a 10 stimuli $20 \mathrm{~Hz}$ train. Values are normalized to the first peak of the train. No significant differences were observed between WT ( $n=14,8$ mice) and APP/PS1 responses ( $n=12,6$ mice; two-way ANOVA, $p=0.7745)$. J, Summary plot of the LTP time course for WT ( $n=12,9$ mice) and APP/PS1 cells ( $n=11,8$ mice). The LTP protocol (100 pulses at a frequency of $100 \mathrm{~Hz}$ repeated three times with a $10 \mathrm{~s}$ inter train interval) was applied at the time indicated by the arrow. Mf-EPSCs were averaged every minute and normalized to the mean amplitude of the 10 min period preceding the LTP induction protocol. $\boldsymbol{K}$, Average values of PTP recorded 5 min after the high-frequency train was applied shows no significant difference between the two genotypes (unpaired $t$ test, $p=0.7373$ ). Values are expressed as percentage of the baseline amplitude of Mf EPSPs. $L$, Mean amplitude of Mf-EPSCs recorded between 30 and 40 min after LTP induction. There is no significant difference in LTP of APP/PS1 compared with WT littermates (Mann-Whitney test, $p=0.7258$ ). Values are expressed as percentage of the baseline Mf-EPSCs amplitude. M, Traces represent Mf-EPSCs obtained at a stimulus frequency of $0.1 \mathrm{~Hz}$ during baseline (10 min), PTP (5 min), LTP (from 30 to 40 min after the train), and LCCG-I (5 min after drug application). All data represented as mean \pm SEM.

a train of 10 stimuli at $20 \mathrm{~Hz}$ (10th EPSC in the train vs first EPSC in the train, WT, $7.2 \pm 0.8$; APP/PS1 7.0 \pm 1.3 ; Fig. $3 H, I$ ).

We further analyzed presynaptic forms of plasticity at MfCA3 synapses in response to high-frequency trains which trigger a short lasting post-tetanic potentiation (PTP) followed by an NMDAR-independent form of LTP (Nicoll and Schmitz, 2005). We used a high-frequency induction protocol consisting of 100 pulses at a frequency of $100 \mathrm{~Hz}$ repeated three times with a $10 \mathrm{~s}$ intertrain interval, in the presence of bicuculline $(10 \mu \mathrm{M}), \mathrm{CGP}-55845(3 \mu \mathrm{M})$ and D-AP5 $(50 \mu \mathrm{M})$ to block $\mathrm{GABA}_{\mathrm{A}}, \mathrm{GABA}_{\mathrm{B}}$, and NMDARs, respectively (Fig. $3 J$ ). In the end of the LTP protocol, bath application of the group II
mGluR agonist LCCG-I decreased the amplitude of EPSCs by $>80 \%$, confirming that these EPSPs were elicited by Mf stimulation (Kirschstein et al., 2004). We compared both the PTP (0-5 min after the train) and LTP levels (30-40 min after the train) in WT and APP/PS1 mice (Fig. $3 K, M$ ). Both genotypes displayed similar levels of PTP (WT 702.5 $\pm 75.1 \%$, and APP/ PS1 $747.7 \pm 112.5 \%$; Fig. $3 K$ ) and LTP (WT $182.9 \pm 23.7 \%$ and APP/PS1 $199.8 \pm 48.7 \%$; Fig. $3 L$ ). Overall, we can conclude that basal properties and conventional forms of presynaptic plasticity at Mf-CA3 PC synapses, are in great part unaltered in APP/PS1 mice at the age of 6 months. 
A

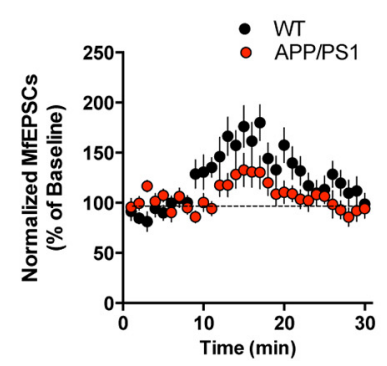

B

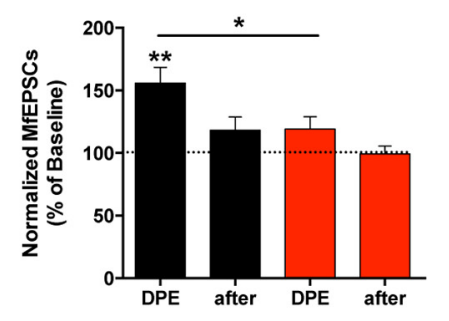

C

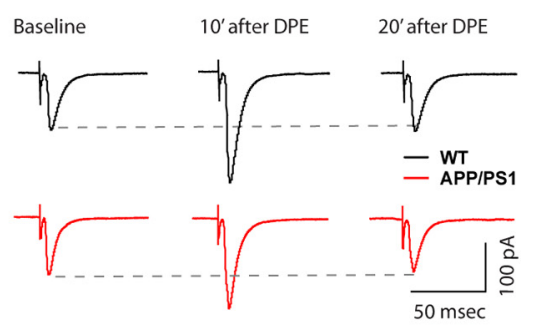

Figure 4. Short-term synaptic plasticity dependent on membrane lipids is strongly reduced at Mf synapses of APP/PS1 mice. $A$, Summary time course of DPE experiments for WT ( $n=10,7$ mice) and APP/PS1 ( $n=12,8$ mice). The DPE protocol consists of a depolarization step from -70 to $-10 \mathrm{mV}$ for $9 \mathrm{~s}$. $\boldsymbol{B}$, Graph summarizing the increase in Mf-EPSCs amplitude after DPE. Values are normalized to the amplitude during the baseline period and data represented as mean \pm SEM. There is an increase in the amplitude of Mf-EPSCs, which is significant in WT mice (single $t$ test compared with baseline, $p=0.0016)$, but not in the APP/PS1 mice ( $p=0.0747)$. C, Sample traces for Mf-EPSCs recorded during baseline (average of 40 sweeps), $5-10$ min after DPE ( 30 sweeps), and 15-20 min after DPE (30 sweeps). All data represented as mean \pm SEM. ${ }^{*} p<0.05,{ }^{* *} p<0.01$.

\begin{abstract}
Short-term synaptic plasticity dependent on membrane lipids is strongly reduced at Mf synapses in APP/PS1 mice

Mf-CA3 synapses were recently shown to express a novel form of short-term synaptic plasticity, namely depolarization-induced potentiation of excitation (DPE) relying on a retrograde signaling mechanism by which endogenously released membrane-derived lipids potentiate synaptic transmission through the inhibition of presynaptic Kv channels (Carta et al., 2014). Because defects in lipid transport and metabolism have been implicated in the pathogenesis of AD (Di Paolo and Kim, 2011), we predicted that DPE may be impaired in APP/PS1 mice. To test this hypothesis, we recorded Mf-CA3 EPSCs before and after a $9 \mathrm{~s}$ long depolarization step in membrane potential (from -70 to $-10 \mathrm{mV}$ ). In WT mice this protocol induced a robust potentiation of Mf-CA3 EPSC amplitude $(155.8 \pm 12.6 \%)$, which lasted several minutes (Fig. $4 A-C$ ). In APP/PS1 mice the amplitude of DPE was strongly diminished $(119.2 \pm 9.7 \%$, two-tailed unpaired $t$ test, $p=0.0297$ between values of DPE; one-sample paired $t$ test shows significant DPE in WT but not in APP/PS1 mice). Importantly, DPE can potentiate AMPA, kainate and NMDA receptor EPSCs, strongly affecting the overall excitatory drive of the network (Carta et al., 2014). Interestingly, DPE affects all Mf synapses onto a single CA3 PCs (Carta et al., 2014), in contrast to synapse-selective forms of synaptic plasticity which we further explored in the APP/PS1 model.
\end{abstract}

\section{LTP of NMDAR-EPSCs is impaired at Mf-CA3 synapses in APP/PS1 mice}

Dysregulation of NMDARs has been proposed as a potential link between APP overexpression, $A \beta$ accumulation and disruption of synaptic function and plasticity (Malinow, 2012). Mf-CA3 synapses were previously thought to only express a presynaptic form of LTP, independent of NMDARs (Nicoll and Schmitz, 2005). Recently it was shown that postsynaptic NMDARs expressed at Mf-CA3 PC synapses themselves undergo LTP contributing to the control of synaptic integration and plasticity (Kwon and Castillo, 2008b; Rebola et al., 2008, 2011; Hunt et al., 2013). We thus tested whether LTP of NMDARs was impaired at Mf-CA3 synapses in APP/PS1 mice. We first recorded AMPAR-EPSCs at $-70 \mathrm{mV}$ at various stimulation frequencies. We subsequently recorded pharmacologically isolated (20 $\mu \mathrm{M}$ NBQX) NMDAR-EPSCs at $+40 \mathrm{mV}$ in the same CA3 PCs. We measured the ratio between Mf-EPSC peak amplitude at $+40 \mathrm{mV}$ (in the presence of NBQX) and $-70 \mathrm{mV}$, NMDAR/ AMPAR ratio, in WT and APP/PS1 mice, at both $0.1 \mathrm{~Hz}$ (data not shown) and $1 \mathrm{~Hz}$ (Fig. 5). We observed that the NMDAR/AMPAR ratio was similar in both genotypes at 0.1 and $1 \mathrm{~Hz}$ frequencies $(1 \mathrm{~Hz}$ :
WT $28.3 \pm 3.3 \%$ and APP/PS1 $29.9 \pm 3.4 \%$; Fig. $5 A, B$ ). We further confirmed these results by calculating the ratio of the peak amplitude (AMPAR component) and the amplitude $40 \mathrm{~ms}$ after the peak (NMDAR component), from the recordings performed at $+40 \mathrm{mV}$ without NBQX (Fig. 5C). Although frequency facilitation of MfNMDAR-EPSCs ( $\sim 5$-fold) appeared smaller compared with frequency facilitation of AMPAR-EPSCs ( $\sim 6$-fold) in adult WT mice (Fig. 3E), the frequency facilitation of Mf-NMDAR-EPSCs was not different between genotypes (WT $4.8 \pm 0.4$ and APP/PS1 $4.0 \pm 0.2$; Fig. $5 D, E)$.

The subunit composition of NMDARs determines its functional properties (Paoletti et al., 2013). Particularly, the identity of the GluN2 subunit impacts on the affinity for glutamate, modulation by glycine, sensitivity to voltage-dependent block by $\mathrm{Mg}^{2+}$, fractional $\mathrm{Ca}^{2+}$ current and channel kinetics (Paoletti et al., 2013). Several studies have ascribed a role for the GluN2B subunit in mediating most of the toxic effects of $A \beta$ oligomers observed in neuronal cultures and acute slices (Rönicke et al., 2011). We tested whether the composition of NMDARs at MfCA3 PC synapses was changed in APP/PS1 mice. At $0.1 \mathrm{~Hz}$ similar decay times of Mf NMDAR-EPSCs were measured in WT $(103.2 \pm 12.5 \mathrm{~ms})$ and 6-month-old APP/PS1 mice (115.6 \pm 12.0 ms; Fig. $5 F)$. The decay time of NMDAR-EPSCs at $1 \mathrm{~Hz}$ stimulation were similar to the ones at $0.1 \mathrm{~Hz}$ and show no difference between genotypes (WT: $97.5 \pm 3.8 \mathrm{~ms}$, APP/PS1: $99.0 \pm 3.6 \mathrm{~ms}$; Fig. $5 G$ ), suggesting no changes in the subunit composition of NMDARs. To further examine putative changes in subunit composition, we used a highly selective, activity-dependent blocker of GluN2B-containing NMDARs (Ro 25-6981; Fischer et al., 1997). NMDAR-EPSCs evoked by Mf stimulation at $0.1 \mathrm{~Hz}$ were recorded at $+40 \mathrm{mV}$ for $8 \mathrm{~min}$ before applying Ro 25-6981 (1 $\mu \mathrm{M})$ and during $40 \mathrm{~min}$ following the application. We observed a progressive reduction of both the amplitude and decay time constants of NMDAR-EPSCs after pharmacological application of the GluN2B antagonist in both genotypes (Fig. $5 H-J$ ). However, there was no difference in the level of inhibition between the two genotypes (WT 28.2 $\pm 9.6 \%$; APP/PS1 $33.1 \pm 9.2 \%$; unpaired $t$ test, $p=0.7144$ ), indicating a similar content in GluN2B. In line with this, we found no difference between WT and APP/PS1 mice in the decay time of NMDAR-EPSCs before $\left(\mathrm{WT}_{\text {baseline }} 86.5 \pm 4.0 \mathrm{~ms}\right.$ and $\mathrm{APP} / \mathrm{PS}_{\text {baseline }} 90.8 \pm 6.0 \mathrm{~ms}$,) and after the application of Ro 25-6981 $\left(\mathrm{WT}_{\text {Ro25-6981 }} 70.8 \pm 1.8 \mathrm{~ms}\right.$ and APP/PS1 $1_{\text {Ro25-6981 }} 77.3$ $\mathrm{ms} \pm 4.2 \mathrm{~ms}$; Fig. $5 I, J)$.

At Mf-CA3 PC synapses, repeated short bursts of Mf stimulation induce robust LTP of NMDAR-EPSCs without affecting 
A
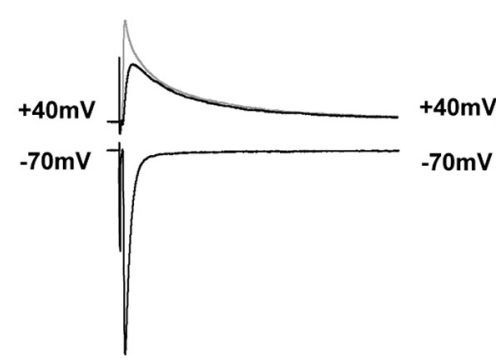

D

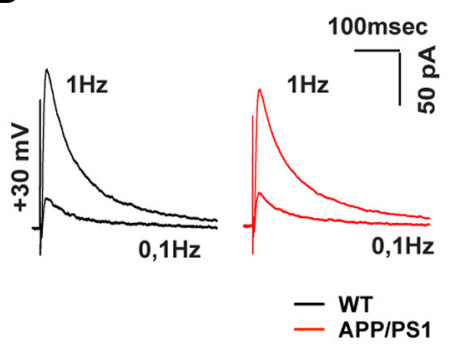

H

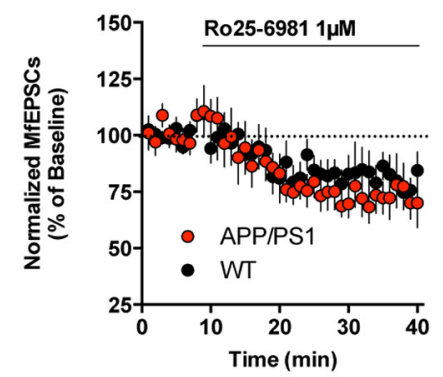

K

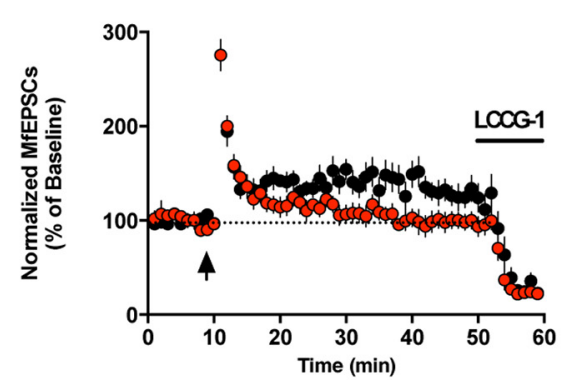

B

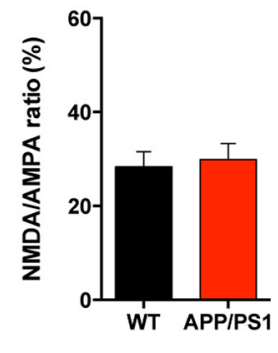

F

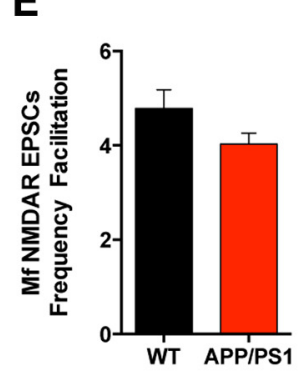

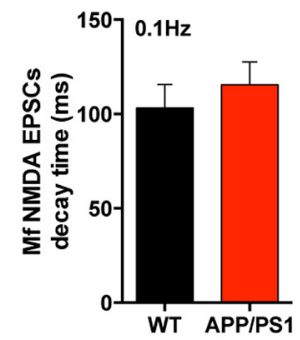

C

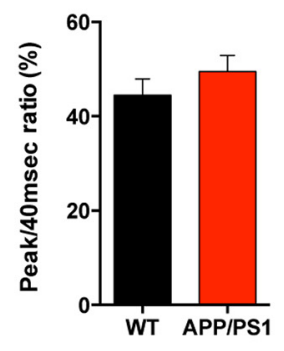

G

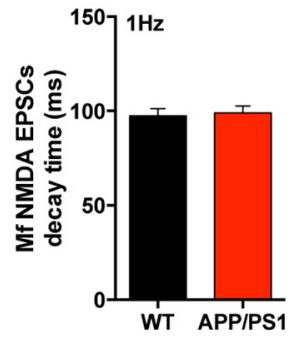

I

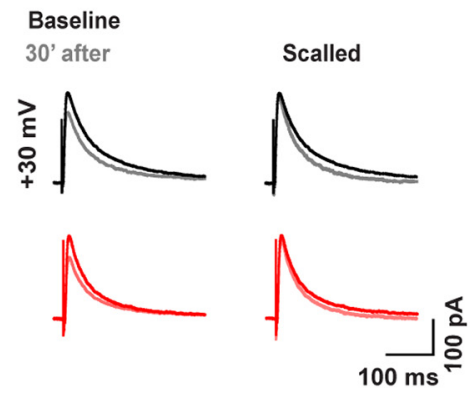

J

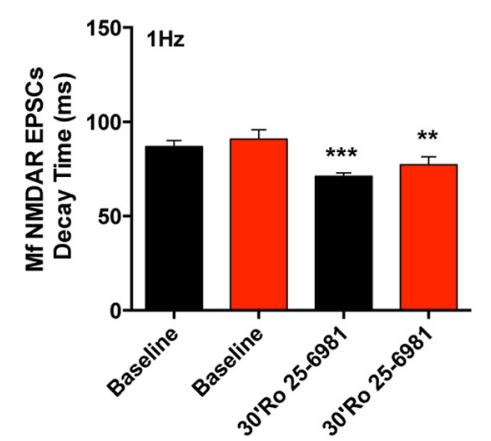

M
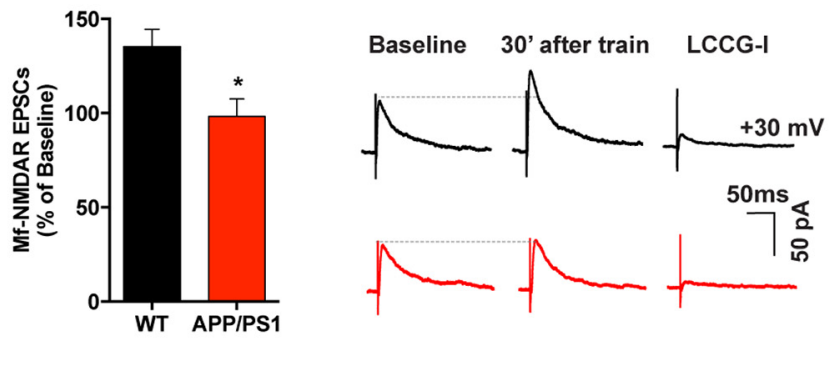

Figure 5. NMDAR-EPSCs and short-term plasticity of NMDAR are maintained in APP/PS1 mice, whereas Mf-NMDAR-dependent LTP is abolished. $A$, Representative traces of Mf-EPSCs recorded at negative $(-70 \mathrm{mV})$ and positive potentials $(+40 \mathrm{mV}$ ), without (lighter trace) and with NBQX (20 $\mu \mathrm{M})$. Bicuculline was present throughout all experiments. $\boldsymbol{B}$, Mean values of NMDAR/AMPAR ratios obtained with isolated currents at $-70 \mathrm{mV}$ (AMPAR) and $+40 \mathrm{mV}$ with NBQX (NMDAR). There is no significant difference between genotypes (WT $n=9,7 \mathrm{mice}$ and APP/PS1, $n=9,6 \mathrm{mice}$; unpaired $t$ test, $p=0.7133)$. C, Bar graph representing mean values obtained for NMDAR/AMPAR ratio performed at $+40 \mathrm{mV}$. The ratio, calculated as the amplitude $40 \mathrm{~ms}$ after the peak divided by the peak amplitude, was not statistically different between the WT ( $44.50 \pm 3.43 \%, n=9,7$ mice) and APP/PS1 cells analyzed (49.49 $\pm 3.42 \%, n=9,6$ mice; unpaired $t$ test, $p=0.3206$ ). $D$, Representative traces of NMDAR-mediated EPSCs recorded at $+40 \mathrm{mV}$, illustrating that both genotypes perform equivalent FF at positive potentials. Traces illustrate average of 40 sweeps obtained at $0.1 \mathrm{~Hz}$ and 60 sweeps at $1 \mathrm{~Hz}$. E, Mean FF values obtained from NMDAR-EPSCs recorded at positive potentials from WT ( $n=29,20$ mice) and APP/PS1 cells $(n=31,22$ mice); unpaired $t$ test, $p=0.1034$. $\boldsymbol{F}$, Mean decay time of Mf-CA3 NMDAR-mediated EPSCs recorded at $0.1 \mathrm{~Hz}$. Decay times are not statistically different between WT ( $n=29,20 \mathrm{mice})$ and APP/PS1 ( $n=31,22$ mice); Mann-Whitney test, $p=0.2099$. G, Same as $\boldsymbol{F}$ but recorded at $1 \mathrm{~Hz}$. Average decay times are also not different (unpaired $t$ test, $p=0.7707$ ) between genotypes. $\boldsymbol{H}$, Time course of Mf-CA3 NMDARs-EPSCs inhibition after application of Ro 25-6981 (1 $\mu \mathrm{M})$. The antagonist was present from the ninth minute until the end of the recordings. Mf NMDAR-mediated EPSCs, obtained every $10 \mathrm{~s}$ $(0.1 \mathrm{~Hz})$ were averaged every minute and inhibition level is expressed as percentage of $8 \mathrm{~min}$ baseline. There is no significant difference between APP/PS1 ( $n=11,9$ mice) and WT ( $n=11,8$ mice, Mann-Whitney test, $p=0.7144$ ). II, Example traces of MfNMDAR-EPSCs obtained at stimulus frequency of $0.1 \mathrm{~Hz}$ during Baseline (8 min), and $30-40$ min of Ro $25-6981$ application (lighter traces). $J$, Mean decay time of Mf-CA3 NMDAR-mediated EPSCs recorded at $0.1 \mathrm{~Hz}$. Average decay times are not statistically different between WT $(n=11,8$ mice) and (Figure legend continues.) 
AMPAR-EPSCs (Kwon and Castillo, 2008b; Rebola et al., 2008). NMDAR-EPSCs evoked by Mf stimulation in CA3 PC were recorded at $+30 \mathrm{mV}$ in the presence of $10 \mu \mathrm{M}$ bicuculline and $3 \mu \mathrm{M}$ CGP-55845 to block GABAergic transmission, and $20 \mu \mathrm{M}$ NBQX to block AMPA and kainate receptors. After establishment of a stable baseline (10 $\mathrm{min}$ ) LTP was induced by six bursts of six stimuli at a frequency of $50 \mathrm{~Hz}$ with a $150 \mathrm{~s}$ inter-burst interval and recorded over a period of $40 \mathrm{~min}$ (Fig. $5 \mathrm{~K}$ ). We found that Mf LTP of NMDAR-EPSCs was significantly reduced in APP/PS1 mice (WT $135.3 \pm 9.2 \%$ and APP/PS1 98.3 $\pm 9.3 \%$; unpaired $t$ test, $p=0.0109$; Fig. $5 L, M)$. Hence, we report that the amount and subunit composition of NMDARs at Mf-CA3 PC synapses is not impacted in the APP/PS1 mouse model of AD. However, we observe a striking impairment in LTP of NMDARs EPSCs at Mf-CA3 PC synapses.

\section{GABAergic transmission and feedforward inhibition are reduced in CA3 PCs of APP/PS1 mice}

Spike transfer between the DG and CA3 is not only controlled by the function and plasticity of excitatory synapses, but also by GABAergic transmission onto CA3 PCs (Rebola et al., 2017). Indeed, monosynaptic innervation of GABAergic interneurons in CA3 via Mf leads to robust feedforward inhibition of CA3-PCs (McBain, 2008; Zucca et al., 2017), which is subject to structural plasticity upon memory encoding (Ruediger et al., 2011; Caroni et al., 2012). Thus, deficits in feedforward inhibition at Mf-CA3 synapses could contribute to early signs of memory impairment in APP/PS1 mice (Kilgore et al., 2010; Viana da Silva et al., 2016).

To test this, we recorded spontaneous IPSCs (sIPSCs) and miniature IPSCs ( mIPSCs) in CA3 PCs. Spontaneous IPSCs were recorded for $10 \mathrm{~min}$ at $-60 \mathrm{mV}$ in the presence of $20 \mu \mathrm{M}$ NBQX and $10 \mathrm{~mm}$ QX-314 added intracellularly to prevent action potential firing in the recorded cell (Fig. 6A-C). We observed that both the distribution of IEIs for sIPSCs (KS test, $p=0.0184$ ) as well as their mean values were statistically different between APP/ PS1 ( $0.38 \pm 0.10 \mathrm{~s})$ and WT mice $(0.19 \pm 0.04 \mathrm{~s}$; Mann-Whitney test, $p=0.0459)$. The distribution of sIPSC amplitudes also differed in APP/PS1 mice, showing a shift toward smaller events (KS test, $p<0.0001)$. Despite this difference in distribution, the mean amplitude values of sIPSCs are not statistically different between the two genotypes (WT $32.4 \pm 3.4 \mathrm{pA}$; APP/PS1 $26.3 \pm 2.0 \mathrm{pA}$ ). The decrease in sIPSC frequency may be caused either by a reduction of the activity of presynaptic interneurons to the recorded CA3 PCs in the slice, or to a change in the number or properties of GABAergic synapses onto CA3 PCs. To address this question, we recorded mIPSCs in the presence of $1 \mu \mathrm{M}$ TTX added to the extracellular medium (Fig. $6 D-F$ ). We observed no significant difference in the distribution and mean IEI of mIPSCs in WT

\footnotetext{
(Figure legend continued.) $\quad$ APP/PS1 mice ( $n=11,9$ mice; repeated-measures two-way ANOVA, $p=0.2196)$. The mean decay time before and after Ro 25-6981 is different for both genotypes (two-way ANOVA, with Sidak's multiple-comparisons test; WT: $p=0.001$; APP/PS1: $p=0.0054$ ), indicating the presence of GluN2B-containing receptors at Mf synapses. $\boldsymbol{K}$, Time course of MF NMDAR-EPSCs in response to NMDAR-LTP protocol. The stimulus train was applied at the time indicated by the arrow and was composed by six bursts of six stimuli given at a frequency of $50 \mathrm{~Hz}$. The six bursts were separated by a $150 \mathrm{~ms}$ interval. Mf NMDAR-mediated EPSCs, evoked every $10 \mathrm{~s}(0.1 \mathrm{~Hz})$ were averaged per minute. $L$, Mean amplitude of Mf NMDARs EPSCs recorded between 30 and 40 min after LTP induction. The Mf-CA3 NMDAR LTP is abolished in the APP/PS1 mice ( $n=11,8$ mice; WT $n=12,9$ mice; unpaired $t$ test, $p=0.0109)$. Values are expressed as percentage of baseline Mf NMDAR-EPSC amplitude. $\boldsymbol{M}$, Representative traces of Mf NMDAR-EPSCS obtained at stimulus frequency of $0.1 \mathrm{~Hz}$ during baseline (10 min), LTP (between $30 \mathrm{~min}$ to $40 \mathrm{~min}$ after the train), and LCCG-I application (5 min after drug treatment). Data presented as mean \pm SEM. ${ }^{*} p<0.05,{ }^{* *} p<0.01,{ }^{* * *} p<0.001$.
}

$(0.95 \pm 0.22 \mathrm{~s})$ and APP/PS1 (1.02 $\pm 0.20 \mathrm{~s})$. Similarly, we found no alteration in the amplitude of mIPSCs (WT $16.9 \pm 0.6 \mathrm{pA}$ and APP/PS1 $17.7 \pm 0.8 \mathrm{pA}$; Fig. $6 F$ ). These results suggest that the decrease in the frequency of spontaneous IPSCs observed in APP/ PS1 mice can be attributed to decreased spiking activity of presynaptic interneurons rather than to a change in the number of GABAergic synapses. However, this does not preclude changes in the properties of GABAergic synaptic transmission or in the intrinsic properties of GABAergic interneurons.

We thus explored whether these changes may translate into changes in feedforward inhibition at Mf-CA3 PC synapses. For this we measured the excitatory/inhibitory ratio of evoked events at Mf-CA3 synapses using different regimes of presynaptic Mf stimulation (Torborg et al., 2010). We first recorded evoked MfEPSCs at $-70 \mathrm{mV}$ in the presence of D-AP5 $(20 \mu \mathrm{M})$ to block NMDARs. We then switched the membrane potential to +10 $\mathrm{mV}$, the reversal potential for cationic ligand-gated channels, to record IPSCs. No significant differences in the ratio were found between APP/PS1 $(0.21 \pm 0.05)$ and WT cells $(0.20 \pm 0.05)$ at 0.1 $\mathrm{Hz}$ (Fig. 6G,H). We further recorded IPSCs evoked by trains of five stimuli at $20 \mathrm{~Hz}$. Similarly, the ratio between evoked EPSCs and IPSCs was not statistically different during the train (Fig. 6I,J), but when we normalized the amplitude of IPSCs in the train to the amplitude of the first IPSC we found that short-term plasticity of inhibition was significantly decreased in APP/PS1 mice compared with WT littermates (two-way ANOVA, $p=0.0478$, WT 2.61 \pm 0.51 and APP/PS1 $1.62 \pm 0.19$; Fig. $6 K$ ). In summary, we observed reduced spontaneous GABAergic inputs onto CA3 PCs, and reduced short term plasticity of feedforward inhibition during $20 \mathrm{~Hz}$ trains of stimulation at Mf-CA3 PC synapses.

\section{Intrinsic properties of CA3 PCs are not altered in 6-month- old APP/PS1 mice}

The intrinsic excitability of neurons shapes not only the rules for synaptic plasticity, but also determines how excitatory and inhibitory inputs are integrated to give rise to an action potential output. We therefore tested whether changes in the integrative properties of CA3 neurons could contribute to our findings of altered synaptic plasticity (e.g., DPE). We performed currentclamp recordings from CA3b PCs in acute hippocampal slices and analyzed various intrinsic properties of these neurons (Fig. $7)$. We found no significant difference in resting membrane potential between WT $(-69.5 \pm 1.7 \mathrm{mV})$ and APP/PS1 CA3 PCs $(-71.3 \pm 1.9 \mathrm{mV}$; Fig. $7 \mathrm{~A})$. Similarly, the input resistance of CA3 PCs was not significantly altered in APP/PS1 mice (WT $356.7 \pm$ 41.7 $\mathrm{M} \Omega$, and APP/PS1 406.8 $\pm 43.2 \mathrm{M} \Omega$; Fig. $7 \mathrm{~B}, C$ ). We then examined the firing threshold and firing frequency of CA3 PCs by injecting current from 0 to $200 \mathrm{pA}$ with $20 \mathrm{pA}$ steps. The firing threshold of CA3 PCs was not significantly altered in APP/PS1 mice (APP/PS1 $-40.0 \pm 1.4 \mathrm{mV})$ compared with WT littermates (WT $-43.0 \pm 1.1 \mathrm{mV}$; Fig. $7 \mathrm{D}$ ). Although there is a tendency for lower firing frequencies in relation to increased current steps in APP/PS1 mice (Fig. 7E), a two-way ANOVA of the two I/O curves did not reveal any significant difference (at the 200 pA step: WT $4.6 \pm 0.8 \mathrm{~Hz}, \mathrm{APP} / \mathrm{PS} 13.4 \pm 0.7 \mathrm{~Hz}$ ). No major differences were found between the two genotypes when the aforementioned recordings were made in the presence of blockers of synaptic transmission: NBQX $(20 \mu \mathrm{M})$, bicuculline $(10 \mu \mathrm{M})$, CGP55845 (3 $\mu \mathrm{M})$, D-AP5 ( $50 \mu \mathrm{M}$; Fig. $7 F-J)$. These findings do not provide evidence that changes in intrinsic excitability could contribute to impaired integration properties of synaptic signals in CA3 PCs. 
A

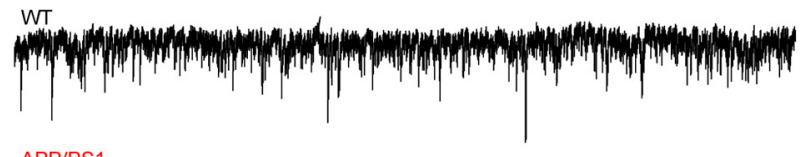

APP/PS1

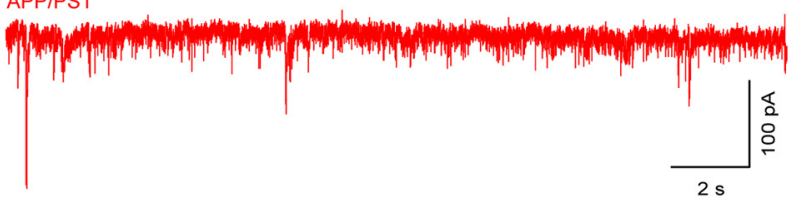

D

\section{WT}

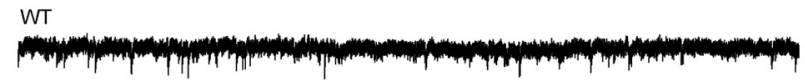

APP/PS1

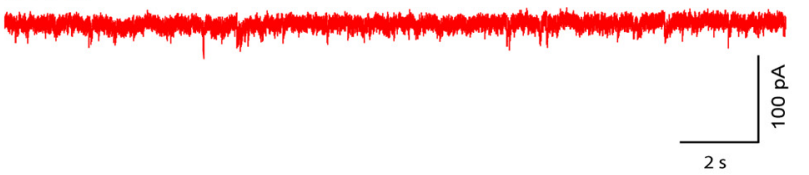

E
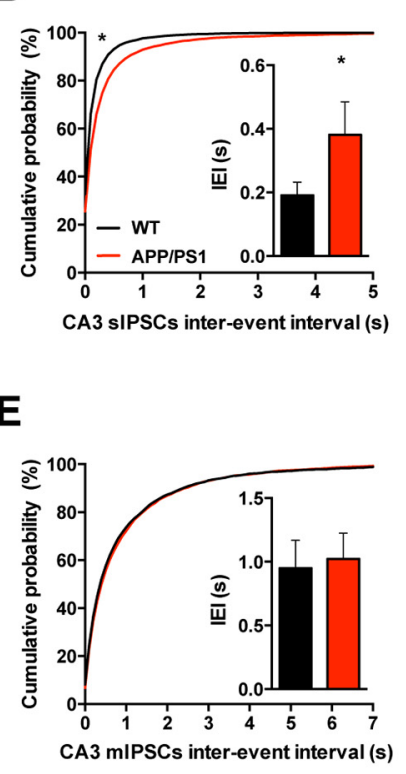

C

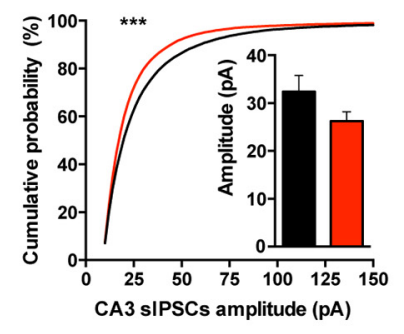

F

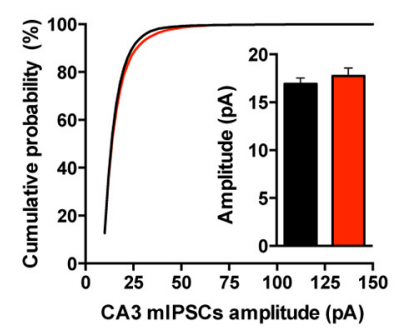

G
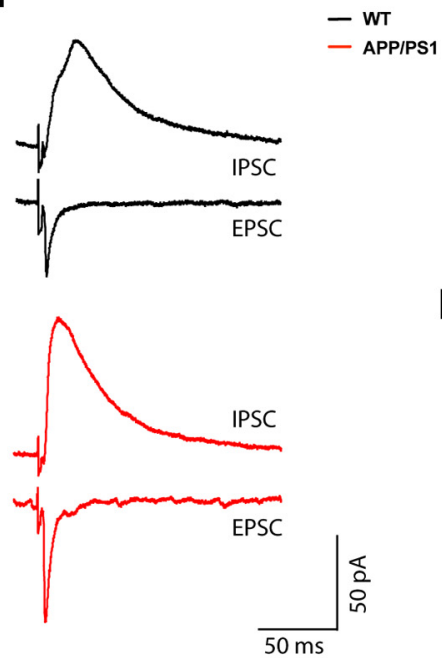

H

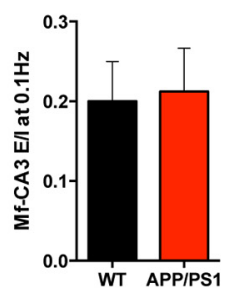

I

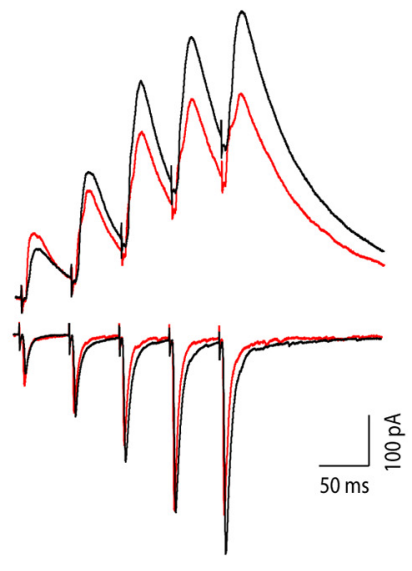

J

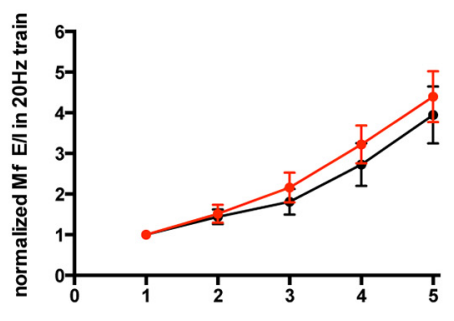

K

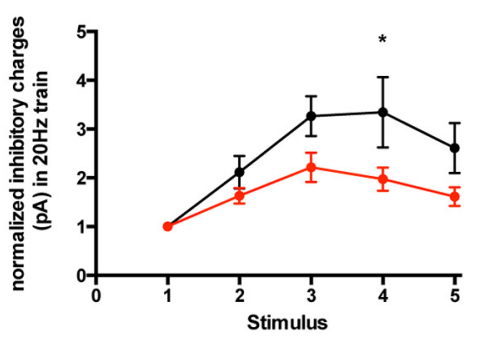

Figure 6. Spontaneous GABAergic transmission and feedforward inhibition are reduced in CA3 PCs of APP/PS1 mice. A, Representative 20-s-long traces of spontaneous IPSCs (20 $\mu \mathrm{M}$ NBQX, extracellularly; $10 \mathrm{~mm}$ QX-314, intracellularly), recorded at $-60 \mathrm{mV}$. B, Mean IEl between sIPSC events recorded during a 10 min period is longer in APP/PS1 cells ( $n=18,6$ mice) compared with WT ( $n=16,6$ mice; Mann-Whitney test, $p=0.0459$ ). The distributions of IEl are also different between genotypes (KS test, $p=0.0194)$. C, There is no significant difference in the mean amplitude values of sIPSCs recorded in WT ( $n=16,6$ mice) and APP/PS1 cells ( $n=18,6$ mice; Mann-Whitney test, $p=0.2494)$, but the distribution of the amplitude values shows a significant shift (KS test, $p>0.0001)$. D. Representative 20 s long traces of spontaneous IPSCs (20 $\mu$ M NBQX and $1 \mathrm{~mm}$ TTX, extracellularly; $10 \mathrm{~mm}$ QX-314, intracellularly) recorded at $-60 \mathrm{mV}$. E, Mean mIPSCS IEI recorded during a 10 min period is not statistically different between WT ( $n=15,6$ mice) and APP/PS1 mice ( $n=15,6$ mice; Mann-Whitney test, $p=0.6752)$ neither is the distribution of IEl values (KS test $=0.2220)$. $\boldsymbol{F}$, Mean mIPSC amplitudes values are not different in the APP/PS1 cells $(n=15,6$ mice) compared with APP/PS1 ones $(n=15,6$ mice; Mann-Whitney test, $p=0.5193)$. The distribution of the amplitude values is also not statistically different between genotypes (KS test, $p=0.1632$ ). G, Representative traces of Mf-EPSCs recorded at negative ( $-70 \mathrm{mV}$ ) and IPSCs recorded at positive potentials $(+10 \mathrm{mV})$ at $0.1 \mathrm{~Hz}$. D-AP5 $(20 \mu \mathrm{m})$ was present through the experiment. $\mathrm{H}, \mathrm{E}-\mathrm{I}$ ratio in CA3 PC of WT and APP/PS1 mice at $0.1 \mathrm{~Hz}$. No significant difference was found between WT cells $(n=16,10$ mice) and APP/PS1 cells $(n=16,10$ mice; Mann-Whitney test, $p=0.9159)$. I, Average of 20 traces illustrating evoked Mf EPSCs (recorded at $-70 \mathrm{mV}$ ) and IPSCs (recorded at $+10 \mathrm{mV}$ ) during a five stimuli $20 \mathrm{~Hz}$ train for WT and APP/PS1 cells. J, Line chart representing the $E-/$ ratio of total charges during the train normalized by the first response. Repeated-measures two-way ANOVA shows no significant difference $(p=0.1712$ ) between WT ( $n=14,9$ mice) and APP/PS1 ( $n=14,9$ mice). $\boldsymbol{K}$, Line chart representing the inhibitory charges during the train in $J$, normalized against the first response. Repeated-measures two-way ANOVA shows significant difference $(p=0.0478)$ between WT ( $n=14,9$ mice) and APP/PS1 cells $(n=$ 14,9 mice). Data presented as mean \pm SEM. ${ }^{*} p<0.01,{ }^{* * *} p<0.001$.

\section{Discussion}

Growing evidence implicates synaptic dysfunction and/or loss, accompanied by neural network reorganization, as the major cause of cognitive impairments in $\mathrm{AD}$, in particular at an early stage of the disease (Selkoe, 2002). However, the precise synaptic phenotypes underlying deficits in memory encoding and retrieval in early-stage $\mathrm{AD}$ pathology are poorly understood. Our previous (Viana da Silva et al., 2016) and present work focus on the investigation of synaptic function and plasticity in CA3 circuits that are crucial for the initial stages of episodic memory encoding and recall (Rebola et al., 2017).

In this study, we characterized the presynaptic and postsynaptic properties of Mf-CA3 synapses in pre-plaque 6-month-old APP/PS1 mice, at a time point when mice already show an impairment in the acquisition of a one-trial memory task (Kilgore et al., 2010; Viana da Silva et al., 2016). Our analysis revealed subtle 
A

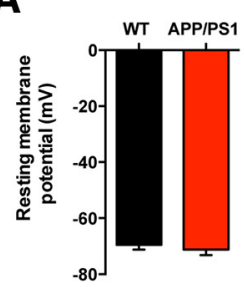

B

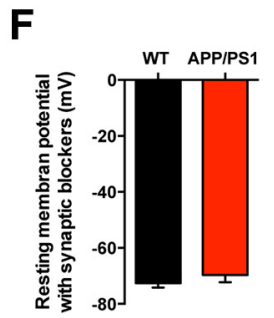

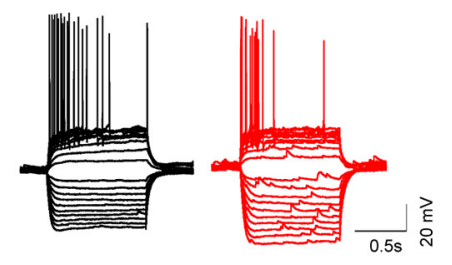

G

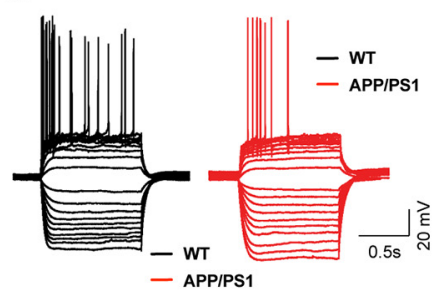

C

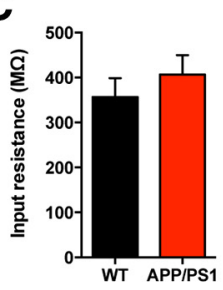

H

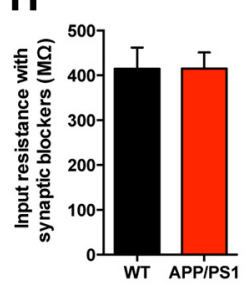

D

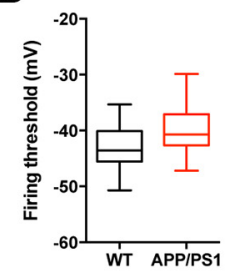

I

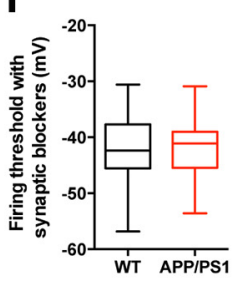

E

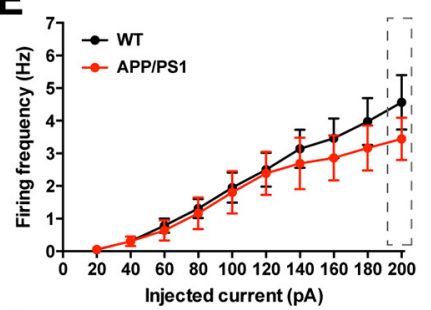

J

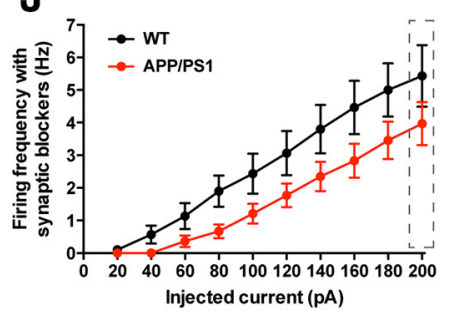

Figure 7. Intrinsic properties of CA3 PCs are not altered in APP/PS1 mice. A, Membrane resting potential measured at the time whole-cell configuration was established. There is no significant difference between WT ( $n=13,4$ mice) and APP/PS1 ( $n=12,4$ mice; unpaired $t$ test, $p=0.5070)$. Data represented as mean \pm SEM. $\boldsymbol{B}$, Representative traces of current-clamp recording during $20 \mathrm{pA} \mathrm{steps} \mathrm{of} \mathrm{increasing} \mathrm{current} \mathrm{injections} \mathrm{for} \mathrm{WT} \mathrm{and} \mathrm{APP/PS1} \mathrm{mice.} \mathrm{C,} \mathrm{There} \mathrm{is} \mathrm{no} \mathrm{significant} \mathrm{difference} \mathrm{in} \mathrm{input} \mathrm{resistance} \mathrm{of} \mathrm{CA3} \mathrm{PC} \mathrm{between} \mathrm{WT} \mathrm{(} n=13,4$ mice) and APP/PS1 ( $n=12,4$ mice; unpaired $t$ test, $p=0.4132$ ). Data represented as mean \pm SEM. $D$, Box plot illustrating the similarity in mean firing threshold of CA3 pyramidal neurons between WT ( $n=13,4$ mice) and APP/PS1 ( $n=12,4$ mice; unpaired $t$ test, $p=0.1005$; whiskers are minimum and maximum values, box represents 25 th and 75 th percentiles, and middle bar represents mean value). $\boldsymbol{E}$, Firing frequency value during increasing current injections; two-way ANOVA, $p=0.5894$ between WT ( $n=13,4$ mice) and APP/PS1 ( $n=12,4$ mice) revealed no significant difference at any current step. Firing frequency is not statistically different when looking exclusively at the last current step ( $200 \mathrm{pA}$; Mann-Whitney test, $p=0.4595)$. Data represented as mean $\pm S E M$. $\boldsymbol{F}-J$, The same experiments as in $\boldsymbol{A}-\boldsymbol{E}$ but with the presence of NBQX (20 $\mu \mathrm{m})$, bicuculline $(10 \mu \mathrm{M})$, CGP $55845(3 \mu \mathrm{m})$, and D-AP5 $(50 \mu \mathrm{m})$. $\boldsymbol{F}$, Bar graph summarizing the lack of significant difference between WT $(-72.60 \pm$ $1.57 \mathrm{mV} ; n=10,4$ mice) and APP/PS1 ( $-69.64 \pm 2.60 \mathrm{mV}, n=11,4$ mice) CA3 PC resting membrane potential (Mann-Whitney test, $p=0.4311)$. Data represented as mean \pm SEM. $\boldsymbol{H}$, Absence of significant difference in the input resistance of CA3 PC between WT ( $414.70 \pm 47.19 \mathrm{M} \Omega ; n=10,4$ mice) and APP/PS1 (415.10 $\pm 35.97 \mathrm{M} \Omega ; n=12,4$ mice; unpaired $t$ test, $p=0.9949)$. Data represented as mean \pm SEM.I, Box plot illustrating the similarity in mean firing threshold of CA3 pyramidal neurons between WT $(-42.26 \pm 2.27 \mathrm{mV} ; n=10,4$ mice) and APP/PS1 ( $-41.42 \pm$ $1.75 \mathrm{mV} ; n=11,4$ mice; unpaired $t$ test, $p=0.7722$; whiskers are min and max values, box represents 25 th and 75 th percentiles, and middle bar represents mean value). $J$, Firing frequency during increasing current injections. Two-way ANOVA ( $p=0.0868)$ shows no significant difference between WT and APP/PS1 cells at any of the steps; firing frequencies recorded at the $200 \mathrm{pA}$ current step are not statistically different in WT ( $5.43 \pm 0.95 \mathrm{~Hz} ; n=10,4$ mice) and APP/PS1 (3.97 $\pm 0.66 ; n=11,4$ mice) when analyzed separately with (unpaired $t$ test, $p=0.2120)$. Data represented as mean \pm SEM.

differences in most morphological features of Mf terminals at both presynaptic and postsynaptic levels. Moreover, we found a robust increase in the number of filopodia, which likely explains the increase in the complexity index specifically of the large MfBs. This observation points to alterations in the feedforward inhibition of this pathway (see section discussing GABAergic synaptic transmission). On the postsynaptic side, we found that the morphological features of ThEs on CA3 PCs dendrites in APP/PS1 mice were comparable to those of WT mice. This lack of major structural changes is consistent with the absence of phenotypes in the basal synaptic properties, such as Mf-EPSC basal amplitude or failure rate. In addition, the conventional forms of presynaptic release properties expressed by MfB, namely short-term facilitation or presynaptic LTP, appear normal in 6-month-old APP/ PS1 mice. These results suggest that early behavioral impairment in this AD mouse model at 6 months (Kilgore et al., 2010; Viana da Silva et al., 2016) are not because of major presynaptic changes at DG-CA3 connections. This is in line with the evidence that presynaptic Mf LTP is not necessary to encode contextual memories (Ruediger et al., 2011). At more advanced stages of amyloid pathology severe impairments of Mf presynaptic LTP develop and might be related to neuroinflammatory processes and more pronounced deficits in behavior (Witton et al., 2010; Maingret et al., 2017).

The principal and surprising finding from our results is that DPE was impaired in 6-month-old APP/PS1 mice. Although the role of DPE in the plasticity of neural circuits in relation to memory encoding and cognitive processes is still unknown, it has been linked to priming Mf-CA3 synapses for LTP (Carta et al., 2014).
The impaired DPE may not be attributed to aberrant intrinsic firing properties of postsynaptic CA3 PCs, because we found no changes in their intrinsic excitability. Instead, it may relate to alterations in lipid metabolism and trafficking (Di Paolo and Kim, 2011). Indeed, recent work in humans identified deficits in lipid metabolism, resulting in reduced arachidonic acid levels in AD patients (Snowden et al., 2017). Importantly, lipid signaling in the brain may potentially modulate both excitatory and inhibitory synapses (García-Morales et al., 2015) and better knowledge about these mechanisms may unravel novel targets to halt or ameliorate $\mathrm{AD}$.

Dysregulation of NMDARs has been proposed to account for defective synaptic plasticity in models of $\mathrm{AD}$ (Malinow, 2012; but see Viana da Silva et al., 2016). This prompted us to investigate possible alterations in the number, composition or conductance of NMDARs. In contrast to previously published findings describing a change in the NMDAR/AMPAR ratio in other cell types and synapses, or in different models of $\mathrm{AD}$ (Roberson et al., 2011), we found that this ratio was not altered at Mf-CA3 synapses. Furthermore, we measured no alteration in the subunit composition of NMDARs after application of Ro 25-6981, suggesting that the levels of synaptic GluN2B-containing NMDARs are not affected, at least not at this early stage of $\mathrm{AD}$. This observation departs from other in vitro studies implicating GluN2Bcontaining NMDARs as particular mediators of neuronal damage in AD (Hu et al., 2009; Rönicke et al., 2011; Ferreira et al., 2012; Cui et al., 2013). Although we found no evidence for changes in the amplitude or subunit composition of Mf synaptic NMDARs in 6-month-old APP/PS1 mice, we examined whether 
the plasticity of NMDARs themselves was affected, a process which has not previously been investigated in the context of AD. Here, we observed that postsynaptic LTP of NMDARs, described at Mf-CA3 synapses (Kwon and Castillo, 2008b; Rebola et al., 2008), is markedly impaired in the APP/PS1 transgenic mice. We cannot rule out that the internal cascades activated by NMDAR activation may be altered, and that the lack of LTP of NMDARs may depend on alterations in postsynaptic signaling. Alteration in postsynaptic signaling may similarly explain deficits in DPE, which is induced postsynaptically (Carta et al., 2014). Previous work of our group has demonstrated that abolition of A/C fiber LTP in CA3 PCs in 6-month-old APP/PS1 mice was caused by activation of upregulated neuronal adenosine $A_{2 A}$ receptors $\left(\mathrm{A}_{2 \mathrm{~A}} \mathrm{Rs}\right)$ in CA3 cells, rather than by dysregulation of NMDAR signaling or altered dendritic spine morphology (Viana da Silva et al., 2016). The mechanisms underlying the loss of LTP of NMDARs at Mf-CA3 synapses may also be related to alterations in $A_{2 A} R$ protein levels or signaling cascades because coactivation of $A_{2 A} R$ and mGluR5 is required for this form of plasticity (Rebola et al., 2008).

Following in vivo studies reporting alterations in GABAergic activity in models of amyloidosis (Verret et al., 2012), we investigated potential alterations in GABAergic synaptic transmissions onto CA3 PCs in these mice. We describe a decrease in the frequency of sIPSCs in APP/PS1 mice (but not mIPSCs), which suggests reduced spiking of presynaptic GABAergic interneurons in CA3 or changes in the properties of GABAergic synaptic transmission. This result is in agreement with in vivo studies showing increased activity in the CA3 region in MCI patients (Yassa et al., 2010). Feedforward inhibition controls the efficacy of information transfer between the DG and CA3 cells (McBain, 2008; Evstratova and Tóth, 2014). We established that short-term plasticity of DG-evoked inhibitory synaptic transmission was impaired during a $20 \mathrm{~Hz}$ train stimulation protocol pointing to a dysfunction in synaptic transmission between MfBs and local interneurons. This is in apparent contradiction with the increased number of filopodia that we have observed in our morphometric analysis. Filopodia are known to make synaptic contacts with CA3 interneurons, and are subject to robust structural plasticity, for instance following a one-trial learning paradigm (Ruediger et al., 2011). It is interesting to note that in APP/ PS1 mice, the increased number of filopodia does not correlate with an increase in feedforward inhibition, suggesting that complex homeostatic mechanisms are involved in the regulation of information transfer at DG-CA3 connections in this mouse model of AD. Further work will be needed to understand how GABAergic transmission in the CA3 region is affected at this time point of disease progression. Additional recordings from identified GABAergic neurons would determine how the intrinsic properties and/or synaptic properties of specific subtypes of interneurons are altered. In line with our results, some studies have reported remodeling of GABAergic neurotransmission in the human AD brain (Limon et al., 2012; Fuhrer et al., 2017) and AD transgenic mouse models (Jo et al., 2014; Wu et al., 2014). Especially, APP/PS1 mice were shown to display spontaneous seizures, which are believed to be associated with an excitation/ inhibition imbalance in the pathology of AD (Ramos et al., 2006; Hazra et al., 2013).

Overall, our work explored the presynaptic and postsynaptic features of hippocampal CA3 synapses in a mouse model of $\mathrm{AD}$, providing new insights into synaptic dysfunction at an early stage of AD. This work shows that long-term potentiation of NMDARs is severely impaired at synapses where this form of plasticity is expressed. Beyond this original demonstration, the main message of this work, together with our previous work on A/C synaptic dysfunction in the same animal model (Viana da Silva et al., 2016), is that synapses are not equally affected by overexpression of mutated forms of APP and PS1 proteins. Our results highlight the importance of studying AD phenotypes for the various specific types of synapses forming the CA3 network. This needs to be taken into account when establishing links between synaptic dysfunction and behavioral impairments and is essential for the development of target strategies for disease amelioration.

\section{References}

Acsády L, Kamondi A, Sík A, Freund T, Buzsáki G (1998) GABAergic cells are the major postsynaptic targets of mossy fibers in the rat hippocampus. J Neurosci 18:3386-3403.

Amaral DG, Dent JA (1981) Development of the mossy fibers of the dentate gyrus: I. A light and electron microscopic study of the mossy fibers and their expansions. J Comp Neurol 195:51-86.

Bednarek E, Caroni P (2011) $\beta$-Adducin is required for stable assembly of new synapses and improved memory upon environmental enrichment. Neuron 69:1132-1146.

Bernier BE, Lacagnina AF, Ayoub A, Shue F, Zemelman BV, Krasne FB, Drew MR (2017) Dentate gyrus contributes to retrieval as well as encoding: evidence from context fear conditioning, recall, and extinction. J Neurosci 37:6359-6371.

Caroni P, Donato F, Muller D (2012) Structural plasticity upon learning: regulation and functions. Nat Rev Neurosci 13:478-490.

Carta M, Lanore F, Rebola N, Szabo Z, Da Silva SV, Lourenço J, Verraes A, Nadler A, Schultz C, Blanchet C, Mulle C (2014) Membrane lipids tune synaptic transmission by direct modulation of presynaptic potassium channels. Neuron 81:787-799.

Cui Z, Feng R, Jacobs S, Duan Y, Wang H, Cao X, Tsien JZ (2013) Increased NR2A:NR2B ratio compresses long-term depression range and constrains long-term memory. Sci Rep 3:1036.

Di Paolo G, Kim TW (2011) Linking lipids to Alzheimer's disease: cholesterol and beyond. Nat Rev Neurosci 12:284-296.

Evstratova A, Tóth K (2014) Information processing and synaptic plasticity at hippocampal mossy fiber terminals. Front Cell Neurosci 8:28.

Ferreira IL, Bajouco LM, Mota SI, Auberson YP, Oliveira CR, Rego AC (2012) Amyloid beta peptide 1-42 disturbs intracellular calcium homeostasis through activation of GluN2B-containing $N$-methyl-D-aspartate receptors in cortical cultures. Cell Calcium 51:95-106.

Fischer G, Mutel V, Trube G, Malherbe P, Kew JN, Mohacsi E, Heitz MP, Kemp JA (1997) Ro 25-6981, a highly potent and selective blocker of $\mathrm{N}$-methyl-D-aspartate receptors containing the NR2B subunit: characterization in vitro. J Pharmacol Exp Ther 283:1285-1292.

Fuhrer TE, Palpagama TH, Waldvogel HJ, Synek BJL, Turner C, Faull RL, Kwakowsky A (2017) Impaired expression of GABA transporters in the human Alzheimer's disease hippocampus, subiculum, entorhinal cortex and superior temporal gyrus. Neuroscience 351:108-118.

Galimberti I, Gogolla N, Alberi S, Santos AF, Muller D, Caroni P (2006) Long-term rearrangements of hippocampal mossy fiber terminal connectivity in the adult regulated by experience. Neuron 50:749-763.

Garcia-Alloza M, Robbins EM, Zhang-Nunes SX, Purcell SM, Betensky RA, Raju S, Prada C, Greenberg SM, Bacskai BJ, Frosch MP (2006) Characterization of amyloid deposition in the APPswe/PS1dE9 mouse model of Alzheimer disease. Neurobiol Dis 24:516-524.

García-Morales V, Montero F, González-Forero D, Rodríguez-Bey G, Gómez-Pérez L, Medialdea-Wandossell MJ, Domínguez-Vías G, GarcíaVerdugo JM, Moreno-López B (2015) Membrane-derived phospholipids control synaptic neurotransmission and plasticity. PLoS Biol 13: e1002153.

Haberl MG, Viana da Silva S, Guest JM, Ginger M, Ghanem A, Mulle C, Oberlaender M, Conzelmann KK, Frick A (2015) An anterograde rabies virus vector for high-resolution large-scale reconstruction of 3D neuron morphology. Brain Struct Funct 220:1369-1379.

Haberl MG, Ginger M, Frick A (2017) Dual anterograde and retrograde viral tracing of reciprocal connectivity. Methods Mol Biol 1538:321-340.

Hazra A, Gu F, Aulakh A, Berridge C, Eriksen JL, Žiburkus J (2013) Inhibitory neuron and hippocampal circuit dysfunction in an aged mouse model of Alzheimer's disease. PLoS One 8:e64318-e64319. 
Henze DA, Card JP, Barrionuevo G, Ben-Ari Y (1997) Large amplitude miniature excitatory postsynaptic currents in hippocampal CA3 pyramidal neurons are of mossy fiber origin. J Neurophysiol 77:1075-1086.

Hu NW, Klyubin I, Anwyl R, Anwy R, Rowan MJ (2009) GluN2B subunitcontaining NMDA receptor antagonists prevent $\mathrm{A} \beta$-mediated synaptic plasticity disruption in vivo. Proc Natl Acad Sci U S A 106:20504-20509.

Hunt DL, Puente N, Grandes P, Castillo PE (2013) Bidirectional NMDA receptor plasticity controls CA3 output and heterosynaptic metaplasticity. Nat Neurosci 16:1049-1059.

Jankowsky JL, Fadale DJ, Anderson J, Xu GM, Gonzales V, Jenkins NA, Copeland NG, Lee MK, Younkin LH, Wagner SL, Younkin SG, Borchelt DR (2004) Mutant presenilins specifically elevate the levels of the 42 residue beta-amyloid peptide in vivo: evidence for augmentation of a 42 -specific gamma secretase. Hum Mol Genet 13:159-170.

Jo S, Yarishkin O, Hwang YJ, Chun YE, Park M, Woo DH, Bae JY, Kim T, Lee J, Chun H, Park HJ, Lee DY, Hong J, Kim HY, Oh SJ, Park SJ, Lee H, Yoon BE, Kim Y, Jeong Y, et al. (2014) GABA from reactive astrocytes impairs memory in mouse models of Alzheimer's disease. Nat Med 20:886-896.

Kesner RP (2013) A process analysis of the CA3 subregion of the hippocampus. Front Cell Neurosci 7:78.

Kesner RP, Rolls ET (2015) A computational theory of hippocampal function, and tests of the theory: new developments. Neurosci Biobehav Rev 48:92-147.

Kilgore M, Miller CA, Fass DM, Hennig KM, Haggarty SJ, Sweatt JD, Rumbaugh G (2010) Inhibitors of class 1 histone deacetylases reverse contextual memory deficits in a mouse model of Alzheimer's disease. Neuropsychopharmacology 35:870-880.

Kirschstein T, von der Brelie C, Steinhäuser M, Vinçon A, Beck H, Dietrich D (2004) L-CCG-I activates group III metabotropic glutamate receptors in the hippocampal CA3 region. Neuropharmacology 47:157-162.

Kwon HB, Castillo PE (2008a) Long-term potentiation selectively expressed by NMDA receptors at hippocampal mossy fiber synapses. Neuron 57 : $108-120$.

Kwon HB, Castillo PE (2008b) Role of glutamate autoreceptors at hippocampal mossy fiber synapses. Neuron 60:1082-1094.

Lanore F, Labrousse VF, Szabo Z, Normand E, Blanchet C, Mulle C (2012) Deficits in morphofunctional maturation of hippocampal mossy fiber synapses in a mouse model of intellectual disability. J Neurosci 32: 17882-17893.

Leutgeb JK, Leutgeb S, Moser MB, Moser EI (2007) Pattern separation in the dentate gyrus and CA3 of the hippocampus. Science 315:961-966.

Limon A, Reyes-Ruiz JM, Miledi R (2012) Loss of functional GABA $A_{A}$ receptors in the Alzheimer diseased brain. Proc Natl Acad Sci U S A 109:10071-10076.

Maingret V, Barthet G, Deforges S, Jiang N, Mulle C, Amédée T (2017) PGE2-EP3 signaling pathway impairs hippocampal presynaptic longterm plasticity in a mouse model of Alzheimer's disease. Neurobiol Aging 50:13-24.

Malinow R (2012) New developments on the role of NMDA receptors in Alzheimer's disease. Curr Opin Neurobiol 22:559-563.

Marchal C, Mulle C (2004) Postnatal maturation of mossy fibre excitatory transmission in mouse CA3 pyramidal cells: a potential role for kainate receptors. J Physiol 561:27-37.

Marchetti C, Marie H (2011) Hippocampal synaptic plasticity in Alzheimer's disease: what have we learned so far from transgenic models? Rev Neurosci 22:373-402.

McBain CJ (2008) Differential mechanisms of transmission and plasticity at mossy fiber synapses. Prog Brain Res 169:225-240.

Nicoll RA, Schmitz D (2005) Synaptic plasticity at hippocampal mossy fibre synapses. Nat Rev Neurosci 6:863-876.

Paoletti P, Bellone C, Zhou Q (2013) NMDA receptor subunit diversity: impact on receptor properties, synaptic plasticity and disease. Nat Rev Neurosci 14:383-400

Pinheiro PS, Perrais D, Coussen F, Barhanin J, Bettler B, Mann JR, Malva JO, Heinemann SF, Mulle C (2007) GluR7 is an essential subunit of presynaptic kainate autoreceptors at hippocampal mossy fiber synapses. Proc Natl Acad Sci U S A 104:12181-12186.

Ramos B, Baglietto-Vargas D, del Rio JC, Moreno-Gonzalez I, Santa-Maria C,
Jimenez S, Caballero C, Lopez-Tellez JF, Khan ZU, Ruano D, Gutierrez A, Vitorica J (2006) Early neuropathology of somatostatin/NPY GABAergic cells in the hippocampus of a PS1 $\times$ APP transgenic model of Alzheimer's disease. Neurobiol Aging 27:1658-1672.

Rebola N, Lujan R, Cunha RA, Mulle C (2008) Adenosine A2A receptors are essential for long-term potentiation of NMDA-EPSCs at hippocampal mossy fiber synapses. Neuron 57:121-134.

Rebola N, Carta M, Lanore F, Blanchet C, Mulle C (2011) NMDA receptordependent metaplasticity at hippocampal mossy fiber synapses. Nat Neurosci 14:691-693.

Rebola N, Carta M, Mulle C (2017) Operation and plasticity of hippocampal CA3 circuits: implications for memory encoding. Nat Rev Neurosci 18:208-220.

Roberson ED, Halabisky B, Yoo JW, Yao J, Chin J, Yan F, Wu T, Hamto P, Devidze N, Yu GQ, Palop JJ, Noebels JL, Mucke L (2011) Amyloid- $\beta$ / Fyn-induced synaptic, network, and cognitive impairments depend on tau levels in multiple mouse models of Alzheimer's disease. J Neurosci 31:700-711.

Rolls ET (2013) A quantitative theory of the functions of the hippocampal CA3 network in memory. Front Cell Neurosci 7:98.

Rönicke R, Mikhaylova M, Rönicke S, Meinhardt J, Schröder UH, Fändrich M, Reiser G, Kreutz MR, Reymann KG (2011) Early neuronal dysfunction by amyloid $\beta$ oligomers depends on activation of NR2B-containing NMDA receptors. Neurobiol Aging 32:2219-2228.

Ruediger S, Vittori C, Bednarek E, Genoud C, Strata P, Sacchetti B, Caroni P (2011) Learning-related feedforward inhibitory connectivity growth required for memory precision. Nature 473:514-518.

Selkoe DJ (2002) Alzheimer's disease is a synaptic failure. Science 298: 789-791.

Snowden SG, Ebshiana AA, Hye A, An Y, Pletnikova O, O’Brien R, Troncoso J, Legido-Quigley C, Thambisetty M (2017) Association between fatty acid metabolism in the brain and Alzheimer disease neuropathology and cognitive performance: a nontargeted metabolomic study. PLoS Med 14:e1002266.

Sperling R (2007) Functional MRI studies of associative encoding in normal aging, mild cognitive impairment, and Alzheimer's disease. Ann N Y Acad Sci 1097:146-155.

Sun Q, Sotayo A, Cazzulino AS, Snyder AM, Denny CA, Siegelbaum SA (2017) Proximodistal heterogeneity of hippocampal CA3 pyramidal neuron intrinsic properties, connectivity, and reactivation during memory recall. Neuron 95:656-672.e3.

Torborg CL, Nakashiba T, Tonegawa S, McBain CJ (2010) Control of CA3 output by feedforward inhibition despite developmental changes in the excitation-inhibition balance. J Neurosci 30:15628-15637.

Verret L, Mann EO, Hang GB, Barth AM, Cobos I, Ho K, Devidze N, Masliah E, Kreitzer AC, Mody I, Mucke L, Palop JJ (2012) Inhibitory interneuron deficit links altered network activity and cognitive dysfunction in Alzheimer model. Cell 149:708-721.

Viana da Silva S, Haberl MG, Zhang P, Bethge P, Lemos C, Gonçalves N, Gorlewicz A, Malezieux M, Gonçalves FQ, Grosjean N, Blanchet C, Frick A, Nägerl UV, Cunha RA, Mulle C (2016) Early synaptic deficits in the APP/PS1 mouse model of Alzheimer's disease involve neuronal adenosine A2A receptors. Nat Commun 7:11915.

Witton J, Brown JT, Jones MW, Randall AD (2010) Altered synaptic plasticity in the mossy fibre pathway of transgenic mice expressing mutant amyloid precursor protein. Mol Brain 3:32.

Wu Z, Guo Z, Gearing M, Chen G (2014) Tonic inhibition in dentate gyrus impairs long-term potentiation and memory in an Alzheimer's [corrected] disease model. Nat Commun 5:4159.

Yassa MA, Stark SM, Bakker A, Albert MS, Gallagher M, Stark CE (2010) High-resolution structural and functional MRI of hippocampal CA3 and dentate gyrus in patients with amnestic mild cognitive impairment. Neuroimage 51:1242-1252.

Zucca S, Griguoli M, Malézieux M, Grosjean N, Carta M, Mulle C (2017) Control of spike transfer at hippocampal mossy fiber synapses in vivo by GABAA and GABAB receptor-mediated inhibition. J Neurosci 37:587598. 